\title{
AN ALGEBRAIC APPROACH TO THE OPENNESS CONJECTURE OF DEMAILLY AND KOLLÁR
}

\author{
MATTIAS JONSSON AND MIRCEA MUSTAŢĂ
}

\begin{abstract}
We reduce the Openness Conjecture of Demailly and Kollár on the singularities of plurisubharmonic functions to a purely algebraic statement.
\end{abstract}

\section{Contents}

1. Introduction 1

2. Background 4

3. Plurisubharmonic functions 13

4. Proof of the main results 21

References 25

\section{INTRODUCTION}

In this paper we study singularities of plurisubharmonic (psh) functions. These are important in complex analytic geometry, see, for instance, Lel69, Sko72, Siu74, Dem87, Kis87, Kis94, DK01. Specifically, we study the openness conjecture of Demailly and Kollár, and reduce this conjecture to a purely algebraic statement.

Let $\varphi$ be the germ of a psh function at a point $x$ on a complex manifold. It is easy to see that the set of real numbers $c>0$ such that $\exp (-c \varphi)$ is locally integrable at $x$ is an interval. It is nonempty by a result of Skoda [Sko72]. The openness conjecture, see [DK01, Remark 5.3], asserts that this interval is open. If we define the complex singularity exponent of $\varphi$ at $x$ by

$$
c_{x}(\varphi)=\sup \{c>0 \mid \exp (-2 c \varphi) \text { is locally integrable at } x\},
$$

then the conjecture can be stated as follows:

Conjecture A. If $c_{x}(\varphi)<\infty$, then the function $\exp \left(-2 c_{x}(\varphi) \varphi\right)$ is not locally integrable at $x$.

Date: October 30, 2018.

Key words and phrases. Plurisubharmonic function, graded sequence, log canonical threshold, valuation.

2010 Mathematics Subject Classification. Primary 32U05; Secondary 32U25, 14F18, 12J20, $14 \mathrm{~B} 05$.

The first author was partially supported by NSF grant DMS-1001740 and the second author was partially supported by NSF grant DMS-1068190. 
In fact, Demailly and Kollár made the following slightly more precise conjecture, which easily implies Conjecture A, see [DK01, Remark 4.4].

Conjecture B. If $c_{x}(\varphi)<\infty$, then for every open neighborhood $U$ of $x$ on which $\varphi$ is defined, we have the estimate

$$
\operatorname{Vol}\left\{y \in U \mid c_{x}(\varphi) \varphi(y)<\log r\right\} \gtrsim r^{2}
$$

as $r \rightarrow 0$.

Here and throughout the paper we write $f(r) \gtrsim r^{2}$ as $r \rightarrow 0$ if there exists $c>0$ such that $f(r) \geq c r^{2}$ for all sufficiently small $r$.

Demailly and Kollár also proved that Conjecture A implies a stronger openness statement, namely, that the local integrability of $\exp (-2 \varphi)$ is an open condition with respect to the $L_{\text {loc }}^{1}$ topology, see [DK01, Conjecture 5.4].

Conjectures A and B are easily verified in dimension one. A proof in the twodimensional case was given in [FJ05b. In higher dimensions, they are open. In this paper we reduce Conjecture B to a purely algebraic conjecture:

Conjecture C. Let $k$ be an algebraically closed field of characteristic zero. Let

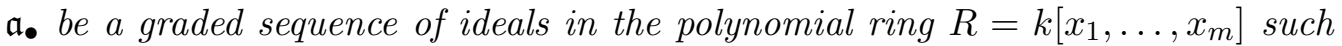
that $\mathfrak{a}_{1}$ is $\mathfrak{m}$-primary, where $\mathfrak{m}$ is a maximal ideal in $R$. Then there exists a quasimonomial valuation $v$ on $R$ which computes $\operatorname{lct}\left(\mathfrak{a}_{\bullet}\right)$.

Let us briefly explain the meaning of the terms here; see $\$ 2.1$ and [JM12 for more details. The $\log$ canonical threshold $\operatorname{lct}(\mathfrak{a})$ of an ideal $\mathfrak{a} \subseteq R$ is the algebrogeometric analogue of the complex singularity exponent. A graded sequence $\mathfrak{a}_{\bullet}=$ $\left(\mathfrak{a}_{j}\right)_{j=1}^{\infty}$ of ideals in $R$ is a sequence such that $\mathfrak{a}_{i} \cdot \mathfrak{a}_{j} \subseteq \mathfrak{a}_{i+j}$ for all $i, j \geq 1$ (we always assume that some $\mathfrak{a}_{i}$ is nonzero). We then define

$$
\operatorname{lct}\left(\mathfrak{a}_{\bullet}\right):=\sup _{j} j \operatorname{lct}\left(\mathfrak{a}_{j}\right)=\lim _{j \rightarrow \infty} j \operatorname{lct}\left(\mathfrak{a}_{j}\right),
$$

where the limit is over those $j$ for which $\mathfrak{a}_{j}$ is nonzero. Similarly, if $v: R \rightarrow \mathbf{R}_{\geq 0}$ is a valuation, then we set

$$
v\left(\mathfrak{a}_{\bullet}\right):=\sup _{j} j^{-1} v\left(\mathfrak{a}_{j}\right)=\lim _{j \rightarrow \infty} j^{-1} v\left(\mathfrak{a}_{j}\right),
$$

where again the limit is over the $j$ for which $\mathfrak{a}_{j}$ is nonzero. One can show that

$$
\operatorname{lct}\left(\mathfrak{a}_{\bullet}\right)=\inf _{v} \frac{A(v)}{v\left(\mathfrak{a}_{\bullet}\right)}
$$

where the infimum is over quasimonomial valuations $v$, that is, valuations that are monomial in suitable coordinates on a suitable blowup of Spec $R$. Here $A(v)$ is the log discrepancy of $v$. Finally, we say that a quasimonomial valuation $v$ computes $\operatorname{lct}\left(\mathfrak{a}_{\bullet}\right)$ if the infimum in $(\star)$ is achieved by $v$.

Conjecture $\mathrm{C}$ holds in dimension two, see [JM12, §9]. In higher dimensions, it is open. Our main result is

Theorem D. If Conjecture $C$ holds for any dimension $m \leq n$ and any algebraically closed field $k$ of characteristic zero, then Conjecture B holds in dimension $n$. 
In fact, we prove a slightly more general result, with the log canonical threshold replaced by more general jumping numbers in the sense of [ELSV04, see Theorem D' in \$3.1. This result has the following consequence. Let $\varphi$ be a psh function on a complex manifold $U$. Recall that the multiplier ideal $\mathcal{J}(\varphi)$ of $\varphi$ is the analytic ideal sheaf on $U$ whose stalk at a point $x$ is given by the set of holomorphic germs $f \in \mathcal{O}_{x}$ such that $|f|^{2} e^{-2 \varphi}$ is locally integrable at $x$. This is a coherent ideal sheaf. Now define $\mathcal{J}^{+}(\varphi)$ as the (increasing, locally stationary) limit of $\mathcal{J}((1+\varepsilon) \varphi)$ as $\varepsilon \searrow 0$. We then show that a suitable generalization of Conjecture $\mathrm{C}$ implies that $\mathcal{J}^{+}(\varphi)=\mathcal{J}(\varphi)$, see Remark 3.10.

We note that one can formulate a version of Conjecture $\mathrm{C}$ in a more general setting, dealing with arbitrary graded sequences on regular excellent connected schemes over Q. It was shown in [JM12] that this more general conjecture follows from the special case in Conjecture $\mathrm{C}$ above.

One can also formulate a similar conjecture for subadditive sequences. Recall that a sequence $\mathfrak{b}_{\bullet}=\left(\mathfrak{b}_{j}\right)_{j=1}^{\infty}$ of nonzero ideals in $R$ is subadditive if $\mathfrak{b}_{i} \cdot \mathfrak{b}_{j} \supseteq \mathfrak{b}_{i+j}$ for all $i, j \geq 1$. As in the case of graded sequences, we can $\operatorname{define} \operatorname{lct}\left(\mathfrak{b}_{\bullet}\right)$ and $v\left(\mathfrak{b}_{\bullet}\right)$ when $v: R \rightarrow \mathbf{R}_{\geq 0}$ is a valuation, and we can consider whether $v$ computes $\operatorname{lct}\left(\mathfrak{b}_{\bullet}\right)$. We say that $\mathfrak{b}_{\bullet}$ has controlled growth if

$$
\frac{1}{j} v\left(\mathfrak{b}_{j}\right) \leq v\left(\mathfrak{b}_{\bullet}\right) \leq \frac{1}{j}\left(v\left(\mathfrak{b}_{j}\right)+A(v)\right)
$$

for all $j \geq 1$ and all quasimonomial valuations $v$ on $R$ (with the left inequality being obvious). Subadditive systems usually arise as multiplier ideals and then are of controlled growth, see Proposition 3.12 and also [JM12, Proposition 2.13]. We show that Conjecture $\mathrm{C}$ implies (in fact, it is equivalent to) the following statement.

Conjecture E. Let $\mathfrak{b}$ • be a subadditive sequence of ideals in an excellent regular domain $R$ of equicharacteristic zero. If $\mathfrak{b}_{\bullet}$ is of controlled growth and there is a maximal ideal $\mathfrak{m}$ in $R$ and a positive integer $p$ such that $\mathfrak{m}^{p j} \subseteq \mathfrak{b}_{j}$ for all $j$, then there exists a quasimonomial valuation $v$ on $R$ such that $v$ computes $\operatorname{lct}\left(\mathfrak{b}_{\bullet}\right)$.

It is this form of the conjecture that we will use in the proof of Theorem D. Let us now indicate the strategy of this proof. Suppose $\varphi$ is a psh germ at a point $x$ on a complex manifold with $\lambda:=c_{x}(\varphi)<\infty$. To $\varphi$ we associate a sequence $\mathfrak{b}_{\bullet}=\left(\mathfrak{b}_{j}\right)_{j \geq 1}$ of ideals by letting $\mathfrak{b}_{j}$ be the analytic multiplier ideal of the psh function $j \varphi$. It follows from [DEL00] that $\mathfrak{b}_{\bullet}$ is subadditive. Further, using techniques due to Demailly [Dem92, Dem93], one can show that $\mathfrak{b}_{\bullet}$ has controlled growth and that the singularities of $\mathfrak{b}_{j}$ closely approximate those of $\varphi$.

The complex singularity exponent $c_{y}(\varphi)$ is a lower semicontinuous function of the point $y$, so we can define $V$ as the "log canonical locus of $\lambda \varphi$ ", that is, the germ at $x$ of the analytic set defined by $c_{y}(\varphi) \leq \lambda$. Assume that $V$ is smooth at $x$ (this is in fact no restriction) and let $\mathcal{O}_{x, V}$ be the localization of the ring of holomorphic germs at $x$, at the prime ideal defined by $V$. Since the latter ring is excellent [Mat80, Theorem 102], so is $\mathcal{O}_{x, V}$.

We now have the subadditive sequence $\mathfrak{b}_{\bullet} \cdot \mathcal{O}_{x, V}$ of ideals in the excellent, regular local ring $\mathcal{O}_{x, V}$. By applying Conjecture E, we conclude that there exists 
a quasimonomial valuation of $\mathcal{O}_{x, V}$ computing $\operatorname{lct}\left(\mathfrak{b}_{\bullet} \cdot \mathcal{O}_{x, V}\right)$. This valuation is monomial in suitable algebraic coordinates on a regular scheme $X$ admitting a projective birational map to $\operatorname{Spec} \mathcal{O}_{x, V}$. We can "analytify" the latter map and interpret the quasimonomial valuation as an analytic invariant, the Kiselman number of $\varphi$. Using basic properties of psh functions and Kiselman numbers we then obtain the desired volume estimates in Conjecture B.

As already mentioned, Conjecture $\mathrm{C}$ holds in dimension two, so we obtain a new proof of the openness conjecture in dimension two. In fact, this proof is quite similar to the one in [FJ05b. The strategy in loc. cit. is to consider a subspace $\mathcal{V}$ of semivaluations $v: \mathcal{O}_{x} \rightarrow[0,+\infty]$ satisfying $v\left(\mathfrak{m}_{x}\right)=1$, where $\mathfrak{m}_{x}$ is the maximal ideal. One can equip $\mathcal{V}$ with a natural topology in which it is compact Hausdorff; it also has the structure of a tree and is studied in detail in [FJ04] (see also [Jon12]). To a psh germ at $x$ one can associate a lower semicontinuous function on $\mathcal{V}$ whose minimum is equal to $c_{x}(\varphi)$. It turns out that the minimum must occur for a semivaluation that is either quasimonomial or associated to the germ of an analytic curve at $x$. In both cases one can deduce the volume estimate in Conjecture B using a simplified version of the arguments in 84.2 .

In higher dimensions, the analogue of the space $\mathcal{V}$ was studied in [BFJ08, where it was shown that $c_{x}(\varphi)$ can be computed using quasimonomial valuations. However, when $\varphi$ does not have an isolated singularity at the origin, it seems difficult to define a suitable lower semicontinuous functional directly on $\mathcal{V}$, having minimum equal to $c_{x}(\varphi)$. The idea is to instead work at a generic point of the log canonical locus of $\lambda \varphi$. This does not quite make sense in the analytic category, and for this reason we pass to algebraic arguments using the subadditive sequence $\mathfrak{b}_{\bullet}$. In the algebraic category, localization arguments work quite well and were extensively used in [JM12].

The idea of studing psh functions using valuations was systematically developed in FJ05a, FJ05b, BFJ08, but appears already in the work of Lelong [Lel69] and Kiselman [Kis87, Kis94]. For some recent work on the singularities of psh functions, see also [Ras06, Ber06, Lag10, Gue10].

The paper is organized as follows. In $\$ 2$ we review facts about sequences of ideals and $\log$ canonical thresholds in an algebraic setting and adapt some of the statements to the setting of complex analytic manifolds. We also prove the equivalence of Conjectures $\mathrm{C}$ and $\mathrm{E}$ above. In 93 we discuss plurisubharmonic functions, Kiselman numbers, multiplier ideal sheaves and the Demailly approximation procedure. Finally, the main results are proved in $\$ 4$.

Acknowledgment. We thank A. Rashkovskii for spotting a mistake in the proof of Theorem D' in an earlier version of the paper. We also thank the referee for a careful reading and several useful remarks.

\section{BACKGROUND}

2.1. Algebraic setting. We start by recalling some basic algebraic facts. For more details we refer to JM12] even though much of what follows is standard material. Let $R$ be an excellent, regular domain of equicharacteristic zero. In the 
cases we will consider, $R$ will be the localization at a prime ideal of the ring of germs of holomorphic functions at a point in a complex manifold.

2.1.1. Quasimonomial valuations. By a valuation on $R$ we mean a rank 1 valuation $v: R \backslash\{0\} \rightarrow \mathbf{R}_{\geq 0}$. A valuation is divisorial if there exists a projective birational morphism $\pi: X \rightarrow \operatorname{Spec} R$, with $X$ regular, a prime divisor $E$ on $X$

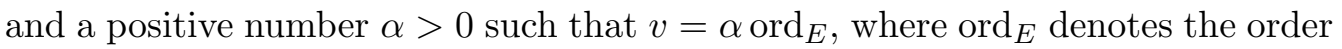
of vanishing along $E$.

More generally, consider a projective birational morphism $\pi: X \rightarrow \operatorname{Spec} R$, with $X$ regular, a reduced simple normal crossing divisor $E=\sum_{i \in I} D_{i}$ on $X$, a subset $J \subseteq I$ such that $\bigcap_{i \in J} D_{i} \neq \emptyset$, an irreducible component $Z$ of $\bigcap_{i \in J} D_{i}$ and nonnegative numbers $\alpha_{i} \geq 0, i \in J$, not all zero. Then there is a unique valuation $v$ on $R$ such that the following holds: if $\left(u_{j}\right)_{j \in J}$ are local coordinates at the generic point $\xi$ of $Z$ such that $D_{j}=\left\{u_{j}=0\right\}$ and we write $f \in R \subseteq \mathcal{O}_{X, \xi} \subseteq \widehat{\mathcal{O}}_{X, \xi}$ as $f=\sum_{\beta} c_{\beta} u^{\beta}$, with $c_{\beta} \in \widehat{\mathcal{O}}_{X, \xi}$ and, for each $\beta$, either $c_{\beta}=0$ or $c_{\beta}(\xi) \neq 0$, then

$$
v(f)=\min \left\{\sum_{i} \alpha_{i} \beta_{i} \mid c_{\beta} \neq 0\right\}
$$

We call such a valuation quasimonomia $\sqrt{1}$ and we say that the morphism $\pi: X \rightarrow$ $\operatorname{Spec} R$ is adapted to $v$. In general, $\pi$ is not unique. On the other hand, we can always choose it so that $\alpha_{i}>0$ for all $i$ and that the $\alpha_{i}$ are rationally independent, see [JM12, Lemma 3.6]. Finally note that quasimonomial valuations are also known as Abhyankar valuations, see [ELS03] and [JM12, §3.2].

2.1.2. Log discrepancy. Using the notation above we define the log discrepancy $A(v)$ of a quasimonomial valuation $v$ by

$$
A(v)=\sum_{j \in J} \alpha_{j}\left(1+\operatorname{ord}_{D_{j}}(K)\right),
$$

where $K=K_{X / \operatorname{Spec} R}$ is the relative canonical divisor. In particular, $A\left(\operatorname{ord}_{D_{j}}\right)=$ $1+\operatorname{ord}_{D_{j}}(K)$. One can show that the log discrepancy of a quasimonomial valuation does not depend on any choices made, see [JM12, §5.1]. Furthermore, one can extend the definition of log discrepancy to arbitrary valuations on $R$; in this case the log discrepancy can be infinite, see [JM12, $\S 5.2]$.

2.1.3. Log canonical thresholds and jumping numbers. If $\mathfrak{a} \subseteq R$ is a proper nonzero ideal, then we define the log canonical threshold of $\mathfrak{a}$ as

$$
\operatorname{lct}(\mathfrak{a})=\inf _{v} \frac{A(v)}{v(\mathfrak{a})}
$$

where the infimum is over all nonzero valuations on $R$ (it is enough to only consider quasimonomial or even divisorial valuations). The quantity $\operatorname{Arn}(\mathfrak{a})=$

\footnotetext{
${ }^{1}$ As opposed to the convention in JM12, we do not consider the trivial valuation, which is identically zero on $R \backslash\{0\}$, to be quasimonomial.
} 
$\operatorname{lct}(\mathfrak{a})^{-1}$ is called the Arnold multiplicity of $\mathfrak{a}$. More generally, if $\mathfrak{q} \subseteq R$ is a nonzero ideal, then we define

$$
\operatorname{lct}^{\mathfrak{q}}(\mathfrak{a})=\inf _{v} \frac{A(v)+v(\mathfrak{q})}{v(\mathfrak{a})} \quad \text { and } \quad \operatorname{Arn}^{\mathfrak{q}}(\mathfrak{a})=\operatorname{lct}^{\mathfrak{q}}(\mathfrak{a})^{-1}
$$

Then $\operatorname{lct}^{\mathfrak{q}}(\mathfrak{a})$ is a jumping number of $\mathfrak{a}$ in the sense of [ELSV04], and all jumping numbers appear in this way. Note that $\operatorname{lct}(\mathfrak{a})=\operatorname{lct}^{R}(\mathfrak{a})$. The infimum in (2.1) (resp. (2.2)) is attained at some divisorial valuation associated to a prime divisor on some log resolution of $\mathfrak{a}($ resp. $\mathfrak{a} \cdot \mathfrak{q})$. We make the convention that if $\mathfrak{a}=0$ or $\mathfrak{a}=R$, then $\operatorname{lct}^{\mathfrak{q}}(\mathfrak{a})=0$ or $\infty$, respectively.

2.1.4. Graded sequences. We now recall the definitions of the asymptotic invariants for graded sequences of ideals. For proofs and details we refer to [JM12, see also [Mus02]. A sequence of ideals $\mathfrak{a}_{\bullet}=\left(\mathfrak{a}_{j}\right)_{j \geq 1}$ is a graded sequence if $\mathfrak{a}_{i} \cdot \mathfrak{a}_{j} \subseteq \mathfrak{a}_{i+j}$ for all $i, j \geq 1$. For example, if $v$ is a valuation on $R$ and $\alpha$ is a positive real number, then by putting $\mathfrak{a}_{j}:=\{f \in R \mid v(f) \geq j \alpha\}$, we obtain a graded sequence in $R$. We refer to [Laz, $\S 10.1]$ for other examples of graded sequences of ideals. We assume that all graded sequences are nonzero in the sense that some $\mathfrak{a}_{j}$ is nonzero.

It follows from the definition that if $\mathfrak{a}_{\boldsymbol{\bullet}}$ is a graded sequence of ideals in $R$ and $v$ is a valuation on $R$, then $v\left(\mathfrak{a}_{i+j}\right) \leq v\left(\mathfrak{a}_{i}\right)+v\left(\mathfrak{a}_{j}\right)$ for all $i, j \geq 1$. By Fekete's Lemma, this subadditivity property implies that

$$
v\left(\mathfrak{a}_{\bullet}\right):=\inf _{j \geq 1} \frac{v\left(\mathfrak{a}_{j}\right)}{j}=\lim _{j \rightarrow \infty} \frac{v\left(\mathfrak{a}_{j}\right)}{j},
$$

where the limit is over those $j$ such that $\mathfrak{a}_{j}$ is nonzero. Similarly, if $\mathfrak{q}$ is a nonzero ideal in $R$, we have

$$
\operatorname{lct}^{\mathfrak{q}}\left(\mathfrak{a}_{\bullet}\right):=\sup _{j \geq 1} j \cdot \operatorname{lct}^{\mathfrak{q}}\left(\mathfrak{a}_{j}\right)=\lim _{j \rightarrow \infty} j \cdot \operatorname{lct}^{\mathfrak{q}}\left(\mathfrak{a}_{j}\right),
$$

where the limit is over those $j$ such that $\mathfrak{a}_{j}$ is nonzero. We also put

$$
\operatorname{Arn}^{\mathfrak{q}}\left(\mathfrak{a}_{\bullet}\right):=\operatorname{lct}^{\mathfrak{q}}\left(\mathfrak{a}_{\bullet}\right)^{-1} \text {. }
$$

The jumping number $\operatorname{lct}^{\mathfrak{q}}\left(\mathfrak{a}_{\mathbf{0}}\right)$ is positive, but may be infinite. One can show (see [JM12, Corolloray 6.9]) that as in the case of one ideal, we have

$$
\operatorname{lct}^{\mathfrak{q}}\left(\mathfrak{a}_{\bullet}\right)=\inf _{v} \frac{A(v)+v(\mathfrak{q})}{v\left(\mathfrak{a}_{\bullet}\right)}
$$

where the infimum is over all nonzero valuations of $R$ (it is enough, in fact, to only consider quasimonomial or even divisorial valuations).

2.1.5. Subadditive sequences. Let us now review the corresponding notions for the case of subadditive sequences, referring for details to [JM12]. A sequence $\mathfrak{b}_{\bullet}=\left(\mathfrak{b}_{j}\right)_{j \geq 0}$ of nonzero ideals in $R$ is called subadditive if $\mathfrak{b}_{i+j} \subseteq \mathfrak{b}_{i} \cdot \mathfrak{b}_{j}$ for all $i, j \geq 0$. This implies that $v\left(\mathfrak{b}_{i+j}\right) \geq v\left(\mathfrak{b}_{i}\right)+v\left(\mathfrak{b}_{j}\right)$ for all valuations $v$ on $R$ and hence that

$$
v\left(\mathfrak{b}_{\bullet}\right):=\sup _{j} \frac{v\left(\mathfrak{b}_{j}\right)}{j}=\lim _{j \rightarrow \infty} \frac{v\left(\mathfrak{b}_{j}\right)}{j} \in \mathbf{R}_{\geq 0} \cup\{+\infty\} .
$$


A subadditive sequence $\mathfrak{b}_{\bullet}$ has controlled growth if

$$
v\left(\mathfrak{b}_{\bullet}\right) \leq \frac{v\left(\mathfrak{b}_{j}\right)}{j}+\frac{A(v)}{j}
$$

for all $j \geq 1$ and all quasimonomial valuation $v$ (in fact, it is enough to only impose this condition for divisorial valuations). In particular, for such $\mathfrak{b}$. we have $v\left(\mathfrak{b}_{\bullet}\right)<\infty$ for every quasimonomial valuation $v$.

For every subadditive system $\mathfrak{b}_{\bullet}$ and every nonzero ideal $\mathfrak{q} \subseteq R$, we define

$$
\operatorname{lct}^{\mathfrak{q}}\left(\mathfrak{b}_{\bullet}\right):=\inf _{j \geq 1} j \cdot \operatorname{lct}^{\mathfrak{q}}\left(\mathfrak{b}_{j}\right)=\lim _{j \rightarrow \infty} j \cdot \operatorname{lct}^{\mathfrak{q}}\left(\mathfrak{b}_{j}\right) .
$$

We also put $\operatorname{Arn}^{\mathfrak{q}}\left(\mathfrak{b}_{\bullet}\right)=\operatorname{lct}^{\mathfrak{q}}\left(\mathfrak{b}_{\bullet}\right)^{-1}$. For every subadditive sequence we have

$$
\operatorname{lct}^{\mathfrak{q}}\left(\mathfrak{b}_{\bullet}\right)=\inf _{v} \frac{A(v)+v(\mathfrak{q})}{v\left(\mathfrak{b}_{\bullet}\right)}
$$

where the infimum is over all valuations $v$ of $R$ with $A(v)<\infty$ (see JJM12, Corollary 6.8]). It is clear from the definition that $\operatorname{lct}^{\mathfrak{q}}\left(\mathfrak{b}_{\bullet}\right)<\infty$ unless $\mathfrak{b}_{j}=R$ for all $j$. Moreover, if $\mathfrak{b}_{\bullet}$ has controlled growth, then $\operatorname{lct}^{\mathfrak{q}}\left(\mathfrak{b}_{\bullet}\right)>0$. Indeed, one can easily see that

$$
\frac{1}{j} \operatorname{Arn}^{\mathfrak{q}}\left(\mathfrak{b}_{j}\right) \leq \operatorname{Arn}^{\mathfrak{q}}\left(\mathfrak{b}_{\bullet}\right) \leq \frac{1}{j} \operatorname{Arn}^{\mathfrak{q}}\left(\mathfrak{b}_{j}\right)+\frac{1}{j}
$$

for all $j \geq 1$, so that $\operatorname{Arn}^{\mathfrak{q}}\left(\mathfrak{b}_{\bullet}\right)<\infty$.

Subadditive sequences arise algebraically as asymptotic multiplier ideals. If $\mathfrak{a} \bullet$ is a graded sequence of ideals in $R$ and if $\mathfrak{b}_{j}=\mathcal{J}\left(\mathfrak{a}_{\bullet}^{j}\right)$ is the asymptotic multiplier ideal of $\mathfrak{a}_{\bullet}$ of exponent $j$, then $\mathfrak{b}_{\bullet}$ is a subadditive sequence of controlled growth (see [JM12, Proposition 2.13]). Furthermore, we have $\operatorname{lct}^{\mathfrak{q}}\left(\mathfrak{a}_{\bullet}\right)=\operatorname{lct}^{\mathfrak{q}}\left(\mathfrak{b}_{\bullet}\right)$ for every nonzero ideal $\mathfrak{q}$, and $v\left(\mathfrak{a}_{\bullet}\right)=v\left(\mathfrak{b}_{\bullet}\right)$ for every valuation $v$ with $A(v)<\infty$ (see [JM12, Proposition 2.14, 6.2]).

2.1.6. Computing jumping numbers of graded sequences. If $\mathfrak{a}_{\bullet}$ is a graded sequence of ideals in $R$ and $\mathfrak{q}$ is a nonzero ideal, then we say that a nonzero valuation $v$ computes $\operatorname{lct}^{\mathfrak{q}}\left(\mathfrak{a}_{\bullet}\right)$ if $v$ achieves the infimum in 2.3 , that is, $\operatorname{lct}^{\mathfrak{q}}\left(\mathfrak{a}_{\bullet}\right)=\frac{A(v)+v(\mathfrak{q})}{v\left(\mathfrak{a}_{\bullet}\right)}$. Note that if $\operatorname{lct}^{\mathfrak{q}}\left(\mathfrak{a}_{\bullet}\right)=\infty$, then $v\left(\mathfrak{a}_{\bullet}\right)=0$ for every $v$; hence every $v$ computes $\operatorname{lct}^{\mathfrak{q}}\left(\mathfrak{a}_{\bullet}\right)$. In what follows we will focus on the case $\operatorname{lct}^{\mathfrak{q}}\left(\mathfrak{a}_{\mathbf{0}}\right)<\infty$; then every valuation $v$ that computes $\operatorname{lct}^{\mathfrak{q}}\left(\mathfrak{a}_{\bullet}\right)$ must satisfy $A(v)<\infty$.

It was shown in [JM12, Theorem 7.3] that for every graded sequence $\mathfrak{a}_{\bullet}$ and every nonzero ideal $\mathfrak{q}$, there is a valuation $v$ on $R$ that computes $\operatorname{lct}^{\mathfrak{q}}\left(\mathfrak{a}_{\bullet}\right)$. One should contrast this with

Conjecture C'. For every excellent regular domain $R$ of equicharacteristic zero, every graded sequence of ideals $\mathfrak{a}_{\bullet}$, and every nonzero ideal $\mathfrak{q}$ in $R$, there is a quasimonomial valuation $v$ on $R$ that computes $\operatorname{lct}^{\mathfrak{q}}\left(\mathfrak{a}_{\bullet}\right)$.

One can also consider the following special case of this conjecture.

Conjecture C". If $k$ is an algebraically closed field of characteristic zero and $R=k\left[x_{1}, \ldots, x_{m}\right]$, then for every nonzero ideal $\mathfrak{q}$ and every graded sequence $\mathfrak{a}_{\bullet}$ of ideals in $R$ such that $\mathfrak{a}_{1} \supseteq \mathfrak{m}^{p}$ for some maximal ideal $\mathfrak{m}$ in $R$ and some $p \geq 1$, there is a quasimonomial valuation $v$ on $R$ that computes $\operatorname{lct}^{\mathfrak{q}}\left(\mathfrak{a}_{\bullet}\right)$. 
Note that when the ideal $\mathfrak{q}$ is equal to $R$, Conjecture C" specializes to Conjecture $\mathrm{C}$ in the Introduction. It is shown ${ }^{2}$ in [JM12, Theorem 7.6] that Conjecture C' holds for rings of dimension $\leq n$ if and only if Conjecture C" holds for rings of dimension $\leq n$. We note that both conjectures are trivially true in dimension one. They are also true in dimension two. A proof, modeled on ideas in [FJ05b] is given in [JM12, $\S 9$ ].

2.1.7. Computing jumping numbers of subadditive sequences. We now turn to the analogous considerations for subadditive sequences. If $\mathfrak{b}_{\bullet}$ is such a sequence, then a valuation $v$ with $A(v)<\infty$ computes $\operatorname{lct}^{\mathfrak{q}}\left(\mathfrak{b}_{\bullet}\right)$ if $v$ achieves the infimum in (2.5). The following conjecture extends Conjecture E from the Introduction to the case of arbitrary jumping numbers.

Conjecture E'. Let $\mathfrak{q}$ be a nonzero ideal and $\mathfrak{b}_{\bullet}$ a subadditive sequence of ideals in an excellent regular domain $R$ of equicharacteristic zero. If $\mathfrak{b}_{\bullet}$ is of controlled growth and there is a maximal ideal $\mathfrak{m}$ in $R$ and a positive integer $p$ such that $\mathfrak{m}^{p j} \subseteq \mathfrak{b}_{j}$ for all $j$, then there exists a quasimonomial valuation $v$ on $R$ such that $v$ computes $\operatorname{lct}^{\mathfrak{q}}\left(\mathfrak{b}_{\bullet}\right)$.

The key requirement in the above conjecture is that the valuation $v$ be quasimonomial. The next proposition shows that if we drop this requirement, we can find a valuation computing the log canonical threshold. This is the analogue of the corresponding result for graded sequences that we have mentioned above.

Proposition 2.1. Under the assumptions in Conjecture E', there is a nonzero valuation $v$ on $R$ with $A(v)<\infty$ which computes $\operatorname{lct}^{\mathfrak{q}}\left(\mathfrak{b}_{\bullet}\right)$.

Remark 2.2. A similar result appears in [Hu12a, see also [Hu12b].

Proof. The argument follows verbatim the proof of [JM12, Thm 7.3], which treated the case of graded sequences $3^{3}$ If $\operatorname{lct}^{\mathfrak{q}}\left(\mathfrak{b}_{\bullet}\right)=\infty$, then the assertion is trivial: we may take $v$ to be any quasimonomial valuation such that $v(\mathfrak{m})=0$, since in this case $v\left(\mathfrak{b}_{\bullet}\right)=0$. Hence, from now on, we may assume that lct ${ }^{\mathfrak{q}}\left(\mathfrak{b}_{\bullet}\right)<\infty$.

By the assumption on $\mathfrak{b}_{\bullet}$, if $v(\mathfrak{m})=0$, then $v\left(\mathfrak{b}_{\bullet}\right)=0$. Therefore we only need to focus on valuations $v$ with $v(\mathfrak{m})>0$, and, after normalizing, we may assume that $v(\mathfrak{m})=1$. Let us fix $\epsilon$ with $0<\epsilon<\operatorname{Arn}^{\mathfrak{q}}\left(\mathfrak{b}_{\bullet}\right)$ and suppose that $\frac{v\left(\mathfrak{b}_{\bullet}\right)}{A(v)+v(\mathfrak{q})}>\epsilon$. For every $j \geq 1$ we have $\mathfrak{m}^{p j} \subseteq \mathfrak{b}_{j}$, hence $v\left(\mathfrak{b}_{j}\right) \leq j p$, and therefore $v\left(\mathfrak{b}_{\bullet}\right) \leq p$. This implies that $A(v) \leq A(v)+v(\mathfrak{q}) \leq M$, where $M=p / \epsilon$. We thus have

$$
\operatorname{lct}^{\mathfrak{q}}\left(\mathfrak{b}_{\bullet}\right)=\inf _{v \in V_{M}} \frac{A(v)+v(\mathfrak{q})}{v\left(\mathfrak{b}_{\bullet}\right)}
$$

where $V_{M}$ is the set of all valuations $v$ with $v(\mathfrak{m})=1$ and $A(v) \leq M$.

The space of all valuations carries a natural topology, and the subspace $V_{M}$ is compact by [JM12, Proposition 5.9]. Moreover, $A$ is a lower semicontinuous

\footnotetext{
${ }^{2}$ For the comparison with JM12, note that a ring $R$ has equicharacteristic zero iff $\operatorname{Spec} R$ is a scheme over $\mathbf{Q}$.

${ }^{3}$ The proof of JM12, Thm 7.3] involves an extra step, the reduction to the case when all ideals are $\mathfrak{m}$-primary, for some maximal ideal $\mathfrak{m}$ in $R$; in our case, we do not have to worry about this step since this is part of the hypothesis.
} 
function on $V_{M}$, while the functions $v \mapsto v(\mathfrak{q})$ and $v \mapsto v\left(\mathfrak{b}_{\bullet}\right)$ are continuous on $V_{M}$ by [JM12, Proposition 5.7, Corollary 6.6] (for the last assertion we make use of the hypothesis that $\mathfrak{b}_{\bullet}$ has controlled growth). It follows that the function $v \mapsto \frac{A(v)+v(\mathfrak{q})}{v\left(\mathfrak{b}_{\bullet}\right)}$ is lower semicontinuous on $V_{M}$; hence it achieves its infimum at some point $v \in V_{M}$.

2.1.8. Equivalence of conjectures. Our main result is that Conjecture C' implies the openness conjecture, see Theorem D' in \$3.1. As a first step, we show that Conjectures C', C" and E' are equivalent.

Proposition 2.3. If one of Conjectures $C^{\prime}, C^{\prime \prime}$, and $E$ ' holds for all rings of dimension $\leq n$, then the other two conjectures hold for such rings.

Proof. As we have already mentioned, [JM12, Theorem 7.6] gives the equivalence of Conjectures C' and C". On the other hand, it is easy to see that if Conjecture E' holds in dimension $\leq n$, then so does Conjecture C". Indeed, let $\mathfrak{q}$ and $\mathfrak{a}_{\bullet}$ be as in Conjecture C", and let $\mathfrak{b}_{j}=\mathcal{J}\left(\mathfrak{a}_{\bullet}^{j}\right)$. In this case $\mathfrak{b}_{\bullet}$ is a subadditive sequence of controlled growth, and for every $j \geq 1$ we have

$$
\mathfrak{m}^{p j} \subseteq \mathfrak{a}_{1}^{j} \subseteq \mathfrak{a}_{j} \subseteq \mathfrak{b}_{j}
$$

Furthermore, if $v$ is a quasimonomial valuation of $R$ which computes $\operatorname{lct}^{\mathfrak{q}}\left(\mathfrak{b}_{\bullet}\right)$, then, since $v\left(\mathfrak{a}_{\bullet}\right)=v\left(\mathfrak{b}_{\bullet}\right)$ and $\operatorname{lct}^{\mathfrak{q}}\left(\mathfrak{a}_{\bullet}\right)=\operatorname{lct}^{\mathfrak{q}}\left(\mathfrak{b}_{\bullet}\right)$, it follows that $v$ computes $\operatorname{lct}^{\mathfrak{q}}\left(\mathfrak{a}_{\bullet}\right)$. Therefore Conjecture $C^{\prime \prime}$ holds in dimension $\leq n$.

We now assume that Conjecture $\mathrm{C}^{\prime}$ holds in dimension $\leq n$, and consider a nonzero $\mathfrak{q}$ and a subadditive sequence $\mathfrak{b}_{\bullet}$ as in Conjecture $\mathrm{E}^{\prime}$, with $\operatorname{dim}(R) \leq n$. We may assume that $\operatorname{lct}^{\mathfrak{q}}\left(\mathfrak{b}_{\bullet}\right)<\infty$, since otherwise the assertion to be proved is trivial (note also that $\operatorname{lct}^{\mathfrak{q}}\left(\mathfrak{b}_{\bullet}\right)>0$ since $\mathfrak{b}_{\bullet}$ has controlled growth). It follows from Proposition 2.1 that there is a nonzero valuation $w$ of $R$ with $A(w)<\infty$ which computes $\operatorname{lct}^{\mathfrak{q}}\left(\mathfrak{b}_{\bullet}\right)$. In particular, $w\left(\mathfrak{b}_{\bullet}\right)$ is finite and positive. If we put $\mathfrak{a}_{j}=\{f \in R \mid w(f) \geq j\}$, then $\mathfrak{a}_{\bullet}$ is a graded sequence of ideals, and by Conjecture $\mathrm{C}^{\prime}$ there is a quasimonomial valuation $v$ on $R$ which $\operatorname{computes~} \operatorname{lct}^{\mathfrak{q}}\left(\mathfrak{a}_{\bullet}\right)$. It is enough to show that in this case $v$ also computes $\operatorname{lct}^{\mathfrak{q}}\left(\mathfrak{b}_{\bullet}\right)$.

It follows easily from the definition of $\mathfrak{a}_{\bullet}$ that $w\left(\mathfrak{a}_{\bullet}\right)=1$ and if $v\left(\mathfrak{a}_{\bullet}\right)=\gamma$, then

$$
\gamma=\inf \{v(f) / w(f) \mid f \in R, w(f)>0\}
$$

(see for example [JM12, Lemma 2.4]). We first deduce that $\operatorname{lct}^{\mathfrak{q}}\left(\mathfrak{a}_{\bullet}\right) \leq A(w)+$ $w(\mathfrak{q})<\infty$, hence $\gamma>0$. Furthermore, we have $\gamma^{-1} v \geq w$. Since $v$ computes $\operatorname{lct}^{\mathfrak{q}}\left(\mathfrak{a}_{\bullet}\right)$, we have

$$
\gamma^{-1}(A(v)+v(\mathfrak{q}))=\frac{A(v)+v(\mathfrak{q})}{v\left(\mathfrak{a}_{\bullet}\right)} \leq \frac{A(w)+w(\mathfrak{q})}{w\left(\mathfrak{a}_{\bullet}\right)}=A(w)+w(\mathfrak{q}) .
$$

On the other hand, using the fact that $\gamma^{-1} v \geq w$, we obtain $\gamma^{-1} v\left(\mathfrak{b}_{\bullet}\right) \geq w\left(\mathfrak{b}_{\bullet}\right)$, and therefore 2.6 gives

$$
\frac{A(v)+v(\mathfrak{q})}{v\left(\mathfrak{b}_{\bullet}\right)} \leq \frac{A(w)+w(\mathfrak{q})}{w\left(\mathfrak{b}_{\bullet}\right)} .
$$

By assumption, $w$ computes $\operatorname{lct}^{\mathfrak{q}}\left(\mathfrak{b}_{\bullet}\right)$, hence we have equality in (2.7), and $v$ also computes $\operatorname{lct}^{\mathfrak{q}}\left(\mathfrak{b}_{\bullet}\right)$. 
Remark 2.4. By running the argument in the proof of Proposition 2.3 with $\mathfrak{q}=R$, we see that Conjectures $\mathrm{C}$ and $\mathrm{E}$ in the Introduction are equivalent, in the sense that one holds for rings of dimension $\leq n$ if and only if the other one does.

2.1.9. A converse to the conjectures. As a partial converse to the (equivalent) conjectures C', C" and E' above we show that any quasimonomial valuation computes some jumping number. This result will not be used in the sequel. In its formulation and proof we freely use terminology from [JM12].

Proposition 2.5. Let $X$ be an excellent, regular, connected, separated scheme over $\mathbf{Q}$ and let $v$ be a quasimonomial valuation on $X$. Then there exists a nonzero ideal $\mathfrak{q}$ on $X$ and a graded sequence $\mathfrak{a}_{\bullet}$ on $X$ such that $v$ computes $\operatorname{lct}^{\mathfrak{q}}\left(\mathfrak{a}_{\bullet}\right)$.

Proof. By [JM12, Thm 7.8] it suffices to find a nonzero ideal $\mathfrak{q}$ such that the following statement holds: for every valuation $w \in \operatorname{Val}_{X}$ such that $w \geq v$ (in the sense that $w(\mathfrak{a}) \geq v(\mathfrak{a})$ for all ideals $\mathfrak{a}$ on $X)$ we have $A(w)+w(\mathfrak{q}) \geq A(v)+v(\mathfrak{q})$. Here $A=A_{X}$ is the log discrepancy with respect to $X$.

After replacing $X$ by an open neighborhood of the center $c_{X}(v)$ of $v$ on $X$ we may assume that $X=\operatorname{Spec} R$ is affine. Since $v$ is quasimonomial, there exists a proper birational morphism $\pi: Y \rightarrow X$, with $Y$ regular, and algebraic local coordinates $y_{1}, \ldots, y_{n}$ at $c_{Y}(v)$ with respect to which $v$ is monomial. Let $E_{i}=\left\{y_{i}=0\right\}, 1 \leq i \leq n$, be the associated prime divisors on $Y$ and pick $N$ large enough so that $N \geq A\left(\operatorname{ord}_{E_{i}}\right)$ for all $i$. Also write $y_{i}=a_{i} / b_{i}$ with $a_{i}, b_{i} \in R$ nonzero.

We claim that the principal ideal $\mathfrak{q}=\left(b_{1} \cdot \ldots \cdot b_{n}\right)^{N}$ does the job. Indeed, suppose $w \in \operatorname{Val}_{X}$ satisfies $w \geq v$. In particular, we then have

$$
w\left(a_{i}\right) \geq v\left(a_{i}\right) \text { and } \quad w\left(b_{i}\right) \geq v\left(b_{i}\right) \text { for all } i .
$$

Since $v$ is monomial in coordinates $y_{1}, \ldots, y_{n}$ we have

$$
A(v)=\sum_{i=1}^{n} v\left(y_{i}\right) A\left(\operatorname{ord}_{E_{i}}\right) .
$$

By the definition of $A(w)$ we also have

$$
A(w) \geq \sum_{i=1}^{n} w\left(y_{i}\right) A\left(\operatorname{ord}_{E_{i}}\right) .
$$

Equations 2.9 and 2.10 and the definition of $\mathfrak{q}$ now imply

$$
\begin{gathered}
A(w)-A(v)+w(\mathfrak{q})-v(\mathfrak{q}) \geq \sum_{i=1}^{n} A\left(\operatorname{ord}_{E_{i}}\right)\left(w\left(y_{i}\right)-v\left(y_{i}\right)\right)+\sum_{i=1}^{n} N\left(w\left(b_{i}\right)-v\left(b_{i}\right)\right) \\
=\sum_{i=1}^{n} A\left(\operatorname{ord}_{E_{i}}\right)\left(w\left(a_{i}\right)-v\left(a_{i}\right)\right)+\sum_{i=1}^{n}\left(N-A\left(\operatorname{ord}_{E_{i}}\right)\right)\left(w\left(b_{i}\right)-v\left(b_{i}\right)\right) \geq 0,
\end{gathered}
$$

where the last inequality follows from $(2.8)$ and the choice of $N$. This completes the proof. 
Remark 2.6. It follows from [JM12, Thm 7.8] that with the choice of $\mathfrak{q}$ above, $v$ also computes $\operatorname{lct}^{\mathfrak{q}}\left(\mathfrak{b}_{\bullet}\right)$ for some subadditive sequence $\mathfrak{b}_{\bullet}$ as well as $\operatorname{lct}^{\mathfrak{q}}\left(\mathfrak{a}_{\bullet}^{\prime}\right)$, where $\mathfrak{a}_{\bullet}^{\prime}$ is the graded sequence defined by $\mathfrak{a}_{j}^{\prime}=\{v \geq j\}$ for $j \geq 1$.

2.2. Analytic setting. Let $U$ be a complex manifold. When talking about open sets in $U$ we always refer to the classical topology unless mentioned otherwise. By an ideal on $U$ we will always mean a coherent analytic ideal sheaf on $U$. For a point $x \in U, \mathcal{O}_{x}$ denotes the ring of germs of holomorphic functions at $x$. Note that $\mathcal{O}_{x}$ is isomorphic to the ring of convergent power series in $n$ variables over C, where $n=\operatorname{dim}(U)$; hence $\mathcal{O}_{x}$ is an excellent regular local ring, see [Mat80, Thm 102].

We denote by $\mathfrak{m}_{x}$ the maximal ideal in $\mathcal{O}_{x}$. By a valuation at $x$ we mean a valuation on $\mathcal{O}_{x}$ in the sense of $\$ 2.1 .1$.

A subadditive sequence of ideals on $U$ is a sequence $\mathfrak{b}_{\bullet}=\left(\mathfrak{b}_{j}\right)_{j=1}^{\infty}$ of everywhere nonzero ideals on $U$ such that $\mathfrak{b}_{i} \cdot \mathfrak{b}_{j} \supseteq \mathfrak{b}_{i+j}$. If $x \in U$, then we write $\mathfrak{b}_{\bullet} \cdot \mathcal{O}_{x}$ for the corresponding subadditive sequence inside $\mathcal{O}_{x}$. We say that $\mathfrak{b}_{\bullet}$ has controlled growth if $\mathfrak{b}_{\bullet} \cdot \mathcal{O}_{x}$ has controlled growth for all $x \in U$.

If $\mathfrak{q}$ is an everywhere nonzero ideal on $U, \mathfrak{b}_{\bullet}$ is a subadditive sequence of ideals on $U$ and $x \in U$, then we define $\operatorname{lct}_{x}^{\mathfrak{q}}\left(\mathfrak{b}_{\bullet}\right):=\operatorname{lct}^{\mathfrak{q} \mathfrak{O} \mathcal{O}_{x}}\left(\mathfrak{b}_{\bullet} \cdot \mathcal{O}_{x}\right)$ and $\operatorname{Arn}_{x}^{\mathfrak{q}}\left(\mathfrak{b}_{\bullet}\right):=$ $\operatorname{lct}_{x}^{\mathfrak{q}}\left(\mathfrak{b}_{\bullet}\right)^{-1}$. Thus we have

$$
\operatorname{Arn}_{x}^{\mathfrak{q}}\left(\mathfrak{b}_{\bullet}\right)=\sup _{v} \frac{v\left(\mathfrak{b}_{\bullet}\right)}{A(v)+v(\mathfrak{q})},
$$

where the supremum is over all (quasimonomial) valuations at $x$ (note that we simply write $v\left(\mathfrak{b}_{\bullet}\right)$ and $v(\mathfrak{q})$ for $v\left(\mathfrak{b}_{\bullet} \cdot \mathcal{O}_{x}\right)$ and $v\left(\mathfrak{q} \cdot \mathcal{O}_{x}\right)$, respectively).

More generally, we shall consider the following situation. Let $V$ be a germ of a complex submanifold at a point $x$ in a complex manifold. Let $\mathcal{O}_{x, V}$ be the localization of $\mathcal{O}_{x}$ along the ideal $I_{V}$. This is an excellent regular local ring with maximal ideal $\mathfrak{m}_{x, V}$. Consider a subadditive system of ideals $\mathfrak{b}$. defined near $x$ and a nonzero ideal $\mathfrak{q} \subseteq \mathcal{O}_{x}$. We set

$$
\operatorname{lct}_{x, V}^{\mathfrak{q}}\left(\mathfrak{b}_{\bullet}\right):=\operatorname{lct}^{\mathfrak{q} \cdot \mathcal{O}_{x, V}}\left(\mathfrak{b}_{\bullet} \cdot \mathcal{O}_{x, V}\right) \text { and } \quad \operatorname{Arn}_{x, V}^{\mathfrak{q}}\left(\mathfrak{b}_{\bullet}\right):=\operatorname{lct}_{x, V}^{\mathfrak{q}}\left(\mathfrak{b}_{\bullet}\right)^{-1}
$$

Then

$$
\operatorname{Arn}_{x, V}^{\mathfrak{q}}\left(\mathfrak{b}_{\bullet}\right)=\sup _{v} \frac{v\left(\mathfrak{b}_{\bullet}\right)}{A(v)+v(\mathfrak{q})}
$$

where the supremum is over all (quasimonomial) valuations of $\mathcal{O}_{x, V}$.

Note that if $V=\{x\}$, then we recover the previous situation.

2.2.1. Analytification of birational morphisms. Let $x, V$ be as above. Consider a projective birational morphism $\pi: X \rightarrow \operatorname{Spec} \mathcal{O}_{x, V}$ of schemes over C, with $X$ regular. We can then analytify $\pi$ as follows $4^{4}$ Since $\pi$ is projective, there exists a closed embedding

$$
X \hookrightarrow \operatorname{Spec} \mathcal{O}_{x, V} \times_{\text {Spec } \mathbf{C}} \mathbf{P}_{\mathbf{C}}^{N}
$$

such that $\pi$ is the restriction of the projection of the right hand side onto $\operatorname{Spec} \mathcal{O}_{x, V}$. Thus $X$ is cut out by finitely many homogeneous equations with

\footnotetext{
${ }^{4}$ The analytification procedure here is ad hoc and not functorial, but nevertheless related to the construction of a complex manifold associated to a smooth complex projective variety.
} 
coefficients in $\mathcal{O}_{x, V}$. These coefficients can be written as $f_{i} / g$, where $f_{i} \in \mathcal{O}_{x}$ and $g \in \mathcal{O}_{x} \backslash I_{V} \cdot \mathcal{O}_{x}$. Let $U$ be a neighborhood of $x$ on which $g$ and the $f_{i}$ are defined. Set $W:=\{g=0\} \subseteq U$. This is a (possibly empty) analytic subset of $U$ that does not contain $V$. After shrinking $U$ we may assume that $W$ is either empty or contains $x$.

We now define a complex manifold

$$
X^{\text {an }} \hookrightarrow(U \backslash W) \times \mathbf{P}^{N}(\mathbf{C})
$$

as the analytic subset cut out by the same equations as in (2.11). Then $X^{\text {an }}$ is a complex manifold and the induced projection $\pi^{\text {an }}: X^{\text {an }} \rightarrow U \backslash W$ is a proper modification. We shall have more to say about this construction later.

Given a point $y \in U \backslash W$ we define in the same way a projective birational morphism $\pi_{y}: X_{y} \rightarrow \operatorname{Spec} \mathcal{O}_{y}$. Namely, $X_{y} \subseteq \operatorname{Spec} \mathcal{O}_{y} \times_{\operatorname{Spec}} \mathbf{C} \mathbf{P}_{\mathbf{C}}^{N}$ is defined by the same homogeneous polynomials as above. After shrinking $U$ and increasing $W$ (but keeping $x \in U$ and $W \nsupseteq V$ ) we may further obtain that if $E \subseteq \pi_{y}^{-1}(V)$ is any prime divisor, then the image $\pi_{y}(E)$ contains $V$. Further, suppose $\mathfrak{b}$ is an ideal on $U$ and that $\pi: X \rightarrow \operatorname{Spec} \mathcal{O}_{x, V}$ as above is a $\log$ resolution of $\mathfrak{b} \cdot \mathcal{O}_{x, V}$. Then, we may assume that the birational morphism $\pi_{y}: X_{y} \rightarrow \operatorname{Spec} \mathcal{O}_{y}$ is a $\log$ resolution of $\mathfrak{b} \cdot \mathcal{O}_{y}$ for all $y \in U \backslash W$. Further, there is a bijection between the set of prime divisors $E$ of $X_{y}$ for which $\operatorname{ord}_{E}\left(\mathfrak{b} \cdot \mathcal{O}_{y}\right)>0$ and the set of prime divisors $E$ of $X$ such that $\operatorname{ord}_{E}\left(\mathfrak{b} \cdot \mathcal{O}_{x, V}\right)>0$.

2.2.2. Log canonical locus. The key to the proof of Theorem D is to localize at the locus where the log canonical threshold is as small as possible. Let $x$ and $V$ be as above and let $\mathfrak{b} \bullet$ (resp. $\mathfrak{q}$ ) be a subadditive system of ideals (resp. a nonzero ideal) defined on some neighborhood $U$ of $x$. Assume that $U$ is small enough that $V$ is a submanifold of $U$.

Lemma 2.7. Assume that $\mathfrak{b}_{\bullet}$ has controlled growth and that

$$
\operatorname{lct}_{y}^{\mathfrak{q}}\left(\mathfrak{b}_{\bullet}\right) \leq \lambda=\operatorname{lct}_{x}^{q}\left(\mathfrak{b}_{\bullet}\right)
$$

for all $y \in V$, where $\lambda \geq 0$. Then $\operatorname{lct}_{x, V}^{\mathfrak{q}}\left(\mathfrak{b}_{\bullet}\right)=\lambda$.

Proof. Let us first prove that $\operatorname{lct}_{x, V}^{\mathfrak{q}}\left(\mathfrak{b}_{\bullet}\right) \geq \lambda$. For this, fix $m \geq 1$ and pick a log resolution $\pi_{m}: X_{m} \rightarrow \operatorname{Spec} \mathcal{O}_{x}$ of the ideal $\left(\mathfrak{q} \mathfrak{b}_{m}\right) \cdot \mathcal{O}_{x}$. After a base change by $\operatorname{Spec} \mathcal{O}_{x, V} \rightarrow \operatorname{Spec} \mathcal{O}_{x}, \pi_{m}$ induces a $\log$ resolution of the ideal $\left(\mathfrak{q} \mathfrak{b}_{m}\right) \cdot \mathcal{O}_{x, V}$. We have

$$
\operatorname{Arn}_{x}^{\mathfrak{q}}\left(\mathfrak{b}_{m}\right)=\max _{E} \frac{\operatorname{ord}_{E}\left(\mathfrak{b}_{m}\right)}{A\left(\operatorname{ord}_{E}\right)+\operatorname{ord}_{E}(\mathfrak{q})}
$$

where the maximum is over the set of prime divisors $E \subseteq X_{m}$ for which $\operatorname{ord}_{E}\left(\mathfrak{b}_{m}\right)>$ 0 . On the other hand $\operatorname{Arn}_{x, V}^{\mathfrak{q}}\left(\mathfrak{b}_{m}\right)$ is given by the same expression, but where the maximum is only over the subset of prime divisors $E$ for which $\pi_{m}(E)$ contains $V$. It is then clear that $\operatorname{Arn}_{x, V}^{\mathfrak{q}}\left(\mathfrak{b}_{m}\right) \leq \operatorname{Arn}_{x}^{\mathfrak{q}}\left(\mathfrak{b}_{m}\right)$. Dividing by $m$ and letting $m \rightarrow \infty$ yields $\operatorname{Arn}_{x, V}^{\mathfrak{q}}\left(\mathfrak{b}_{\bullet}\right) \leq \operatorname{Arn}_{x}^{\mathfrak{q}}\left(\mathfrak{b}_{\bullet}\right)$ and hence $\operatorname{lct}_{x, V}^{\mathfrak{q}}\left(\mathfrak{b}_{\bullet}\right) \geq \operatorname{lct}_{x}^{\mathfrak{q}}\left(\mathfrak{b}_{\bullet}\right)=\lambda$.

Now we prove the reverse inequality. Pick any $m \geq 1$. Consider a log resolution $\pi_{m}: X_{m} \rightarrow \operatorname{Spec} \mathcal{O}_{x, V}$ of the ideal $\mathfrak{q} \mathfrak{b}_{m} \cdot \mathcal{O}_{x, V}$. As in $\$ 2.2 .1$ this gives rise to an open neighborhood $U_{m}$ of $x$, an analytic subset $W_{m} \subseteq U_{m}$ not containing 
$V \cap U_{m}$, and for each $y \in V \cap\left(U_{m} \backslash W_{m}\right)$, a $\log$ resolution $\pi_{m, y}: X_{m, y} \rightarrow \operatorname{Spec} \mathcal{O}_{y}$ of $\mathfrak{q} \mathfrak{b}_{m} \cdot \mathcal{O}_{y}$. Further, there is a bijection between the set of prime divisors $E \subseteq$ $X_{m, y}$ such that $\operatorname{ord}_{E}\left(\mathfrak{b}_{m} \cdot \mathcal{O}_{y}\right)>0$ and the set of prime divisors $E \subset X_{m}$ for which $\operatorname{ord}_{E}\left(\mathfrak{b}_{m} \cdot \mathcal{O}_{x, V}\right)>0$. This implies that $\operatorname{Arn}_{y}^{\mathfrak{q}}\left(\mathfrak{b}_{m}\right)=\operatorname{Arn}_{x, V}^{\mathfrak{q}}\left(\mathfrak{b}_{m}\right)$ for any $y \in\left(U_{m} \backslash W_{m}\right) \cap V$ since both quantities are calculated using the same divisors. Thus we have

$$
\operatorname{Arn}_{y}^{\mathfrak{q}}\left(\mathfrak{b}_{m}\right)=\operatorname{Arn}_{x, V}^{\mathfrak{q}}\left(\mathfrak{b}_{m}\right) \leq m \operatorname{Arn}_{x, V}^{\mathfrak{q}}\left(\mathfrak{b}_{\bullet}\right)
$$

the inequality being definitional.

Now, for any $y \in\left(U_{m} \backslash W_{m}\right) \cap V$ and any $j \geq 1$ there exists a quasimonomial (or even divisorial) valuation $v_{j}$ at $y$ such that

$$
\frac{v_{j}\left(\mathfrak{b}_{\bullet}\right)}{A\left(v_{j}\right)+v_{j}(\mathfrak{q})} \geq \operatorname{Arn}_{y}^{\mathfrak{q}}\left(\mathfrak{b}_{\bullet}\right)-\frac{1}{j} \text {. }
$$

This gives

$$
\begin{aligned}
& \lambda^{-1} \leq \operatorname{Arn}_{y}^{\mathfrak{q}}\left(\mathfrak{b}_{\bullet}\right) \leq \frac{v_{j}\left(\mathfrak{b}_{\bullet}\right)}{A\left(v_{j}\right)+v_{j}(\mathfrak{q})}+\frac{1}{j} \leq \frac{v_{j}\left(\mathfrak{b}_{m}\right)+A\left(v_{j}\right)}{m\left(A\left(v_{j}\right)+v_{j}(\mathfrak{q})\right)}+\frac{1}{j} \\
& \leq \frac{v_{j}\left(\mathfrak{b}_{m}\right)}{m\left(A\left(v_{j}\right)+v_{j}(\mathfrak{q})\right)}+\frac{1}{m}+\frac{1}{j} \leq \frac{\operatorname{Arn}_{y}^{\mathfrak{q}}\left(\mathfrak{b}_{m}\right)}{m}+\frac{1}{m}+\frac{1}{j} \leq \operatorname{Arn}_{x, V}^{\mathfrak{q}}\left(\mathfrak{b}_{\bullet}\right)+\frac{1}{m}+\frac{1}{j},
\end{aligned}
$$

where the second inequality follows from the assumption that $\mathfrak{b}_{\bullet} \cdot \mathcal{O}_{y}$ has controlled growth. Letting first $j \rightarrow \infty$ and then $m \rightarrow \infty$ we obtain $\operatorname{Arn}_{x, V}^{\mathfrak{q}}\left(\mathfrak{b}_{\bullet}\right) \geq \lambda^{-1}$, and hence $\operatorname{lct}_{x, V}^{\mathfrak{q}}\left(\mathfrak{b}_{\bullet}\right) \leq \lambda$. This completes the proof.

\section{Plurisubharmonic functions}

Let $U$ be a complex manifold. A function $\varphi: U \rightarrow \mathbf{R} \cup\{-\infty\}$ is plurisubharmonic (psh) if $\varphi \not \equiv-\infty$ on any connected component of $U, \varphi$ is upper semicontinuous, and $\tau^{*} \varphi$ is subharmonic or $\equiv-\infty$ for every holomorphic map $\tau: \mathbf{D} \rightarrow U$, where $\mathbf{D} \subseteq \mathbf{C}$ is the unit disc. A germ of a psh function at a point is defined in the obvious way.

A basic example of a psh function is $\varphi=\log \max _{i}\left|f_{i}\right|$, where $f_{1}, \ldots, f_{m}$ are holomorphic functions on $U$. If $x \in U$ and $\mathfrak{q} \subseteq \mathcal{O}_{x}$ is an ideal, then we can define a psh germ $\log |\mathfrak{q}|$ at $x$ by setting

$$
\log |\mathfrak{q}|:=\log \max _{i}\left|f_{i}\right|
$$

where $f_{i} \in \mathcal{O}_{x}$ are generators of $\mathfrak{q}$. The choice of generators only affects $\log |\mathfrak{q}|$ up to a bounded additive term. If $\mathfrak{a}$ and $\mathfrak{b}$ are two ideals in $\mathcal{O}_{x}$, then

$$
\log |\mathfrak{a} \cdot \mathfrak{b}|=\log |\mathfrak{a}|+\log |\mathfrak{b}|+O(1) .
$$

For further facts about psh functions, see e.g. [Hor94, Dem].

3.1. Jumping numbers and singularity exponents. Let $\varphi$ be a psh germ at a point $x$ on a complex manifold and let $\mathfrak{q} \subseteq \mathcal{O}_{x}$ be a nonzero ideal. Define

$$
c_{x}^{\mathfrak{q}}(\varphi)=\sup \left\{c>\left.0|| \mathfrak{q}\right|^{2} \exp (-2 c \varphi) \text { is locally integrable at } x\right\} .
$$

This definition does not depend on the choice of generators used to define $|\mathfrak{q}|$. We also write $c_{x}(\varphi):=c_{x}^{\mathcal{O}_{x}}(\varphi)$. In [DK01], $c_{x}(\varphi)$ is called the complex singularity 
exponent of $\varphi$ at $x$, whereas $c_{x}^{\mathfrak{q}}(\varphi)$ is a jumping number in the sense of [ELSV04]. If $\mathfrak{a} \subseteq \mathcal{O}_{x}$ is a nonzero ideal, then we have

$$
c_{x}^{\mathfrak{q}}(\log |\mathfrak{a}|)=\operatorname{lct}_{x}^{\mathfrak{q}}(\mathfrak{a}),
$$

where the right hand side is defined as in $\S 2.2$, see [DK01, Proposition 1.7] $!^{5} \mathrm{We}$ have the following generalizations of Conjectures $\mathrm{A}$ and $\mathrm{B}$.

Conjecture $\mathbf{A}^{\prime}$. If $c_{x}^{\mathfrak{q}}(\varphi)<\infty$, then the function $|\mathfrak{q}|^{2} \exp \left(-2 c_{x}^{\mathfrak{q}}(\varphi) \varphi\right)$ is not locally integrable at $x$.

This conjecture, which is also due to Demailly and Kollár, can be paraphrased as a semicontinuity statement for multiplier ideals, see Remark 3.10 .

Conjecture B'. If $c_{x}^{\mathfrak{q}}(\varphi)<\infty$, then for any open neighborhood $U$ of $x$ on which $\varphi$ and $\mathfrak{q}$ are defined, we have

$$
\operatorname{Vol}\left\{y \in U\left|c_{x}(\varphi) \varphi(y)-\log \right| \mathfrak{q} \mid<\log r\right\} \gtrsim r^{2}
$$

as $r \rightarrow 0$.

It is clear that Conjecture B' implies Conjecture A' and that neither conjecture depends on the choice of generators of $\mathfrak{q}$. The following result is a variation of Theorem D from the introduction. We shall prove both these theorems in $\$ 4$.

Theorem D'. If Conjecture $C$ " in $\$ 2.1 .6$ holds for all $m \leq n$ and all algebraically closed fields $k$ of characteristic zero, then Conjecture B' holds on complex manifolds of dimension $n$.

3.2. Kiselman numbers. We now recall an analytic version of monomial valuations due to Kiselman Kis87, Kis94. It is a special case of the generalized Lelong numbers introduced by Demailly [Dem87]. As our setting differs slightly from the above references, we give some details for the convenience of the reader.

Let $\Omega$ be a complex manifold of dimension $n, Z \subseteq \Omega$ a connected submanifold of codimension $m \geq 1$ and $D_{1}, \ldots, D_{m}$ distinct, smooth, connected hypersurfaces in $\Omega$ such that $Z=\bigcap_{i} D_{i}$ and such that the $D_{i}$ meet transversely along $Z$. Also suppose we are given positive real numbers $\alpha_{i}>0,1 \leq i \leq m$. In this situation we will associate to any psh function $\varphi$ on $U$ its $\operatorname{Kiselman}$ number $\tau_{Z, D, \alpha}(\varphi) \geq 0$.

In preparation for the definition, pick a point $z \in Z$ and local analytic coordinates $\left(u_{1}, \ldots, u_{n}\right)$ at $z$ such that $D_{i}=\left\{u_{i}=0\right\}$ for $1 \leq i \leq m$ locally at $z$. For $t \in \mathbf{R}_{\leq 0}^{n}$ with $t_{i} \ll 0$ let $D_{u}(t) \subseteq \Omega$ be the polydisc with radius $e^{t}$ and $S_{u}(t)$ its distinguished boundary, that is

$$
D_{u}(t):=\bigcap_{i=1}^{n}\left\{\left|u_{i}\right| \leq e^{t_{i}}\right\} \quad \text { and } \quad S_{u}(t):=\bigcap_{i=1}^{n}\left\{\left|u_{i}\right|=e^{t_{i}}\right\} .
$$

We also write $D_{u}(s)=D_{u}(s, s, \ldots, s)$ for $s \in \mathbf{R}_{\leq 0}$.

Let $\varphi$ be a psh germ at $z$ and pick $\varepsilon>0$ small enough that $\varphi$ is defined in an open neighborhood of the polydisc $D_{u}(\log \varepsilon)$. For $t \in \mathbf{R}_{\leq \log \varepsilon}^{n}$ set

$$
H(t):=\sup _{D_{u}(t)} \varphi=\sup _{S_{u}(t)} \varphi .
$$

\footnotetext{
${ }^{5}$ In loc. cit. only the case $\mathfrak{q}=\mathcal{O}_{x}$ is treated but the same proof works in the general case.
} 
Clearly $H$ is increasing in each argument and it is finite-valued since $\varphi$ is upper semicontinuous. Less obvious is the fact that $H$ is convex; for this see Kis94, p.12]. Note that $H$ is continuous on the closed set $\mathbf{R}_{\leq \log \varepsilon}^{n}$ since it is defined and convex on an open neighborhood of this set.

We now define a new function

$$
h=h_{\varphi, Z, D, z, u, \varepsilon}: \mathbf{R}_{\geq 0}^{n} \rightarrow \mathbf{R}
$$

by setting

$$
h(\alpha):=\lim _{s \rightarrow-\infty} \frac{H(\log \varepsilon+s \alpha)}{s} .
$$

The limit is well defined by the convexity of $H$.

Lemma 3.1. The function $h=h_{\varphi, Z, D, z, u, \varepsilon}$ has the following properties:

(i) $h$ is nonnegative, continuous, concave, 1-homogeneous and increasing in each argument;

(ii) $h$ does not depend on the choice of $\varepsilon$ as long as $\varphi$ is defined in an open neighborhood of $D(\log \varepsilon)$.

If, further, $\alpha_{i}=0$ for $i>m$, then

(iii) $h$ does not depend on the choice of local coordinates $\left(u_{1}, \ldots, u_{n}\right)$ at $z$, as long as $D_{i}=\left\{u_{i}=0\right\}$ for $i \leq m$;

(iv) $h$ does not depend on the choice of point $z \in Z$ as long as $\varphi$ is defined in a neighborhood of $z$.

Proof. To alleviate notation, we shall only write out the relevant part of the subscripts of $h=h_{\varphi, Z, D, z, u, \varepsilon}$.

The fact that $h$ is nonnegative, continuous, concave and increasing follows from $H$ being continuous, convex and increasing. That $h$ is 1-homogeneous is clear. This proves (i).

As for (ii), suppose $0<\varepsilon^{\prime}<\varepsilon$. It is clear that $h_{\varepsilon^{\prime}} \geq h_{\varepsilon}$ since $H$ is increasing. To prove the reverse inequality, first suppose that $\alpha_{i}>0$ for $1 \leq i \leq n$ and set $\delta=\min _{i} \alpha_{i}$. Then

$$
D_{u}(\log \varepsilon+s \alpha) \subseteq D_{u}\left(\log \varepsilon^{\prime}+\left(s+\frac{1}{\delta} \log \frac{\varepsilon}{\varepsilon^{\prime}}\right) \alpha\right),
$$

so that $H(\log \varepsilon+s \alpha) \leq H\left(\log \varepsilon^{\prime}+\left(s+\frac{1}{\delta} \log \frac{\varepsilon}{\varepsilon^{\prime}}\right) \alpha\right)$ for any $s \leq 0$. This implies $h_{\varepsilon}(\alpha) \geq h_{\varepsilon}(\alpha)$. By continuity of $h_{\varepsilon}$ and $h_{\varepsilon^{\prime}}$ we get $h_{\varepsilon} \geq h_{\varepsilon^{\prime}}$ and hence $h_{\varepsilon}=h_{\varepsilon^{\prime}}$ on $\mathbf{R}_{>0}^{n}$.

Now we turn to (iii) and (iv) so suppose $\alpha_{i}=0$ for $i>m$.

Let $\left(u_{1}^{\prime}, \ldots, u_{n}^{\prime}\right)$ be another set of local analytic coordinates at $z$ such that $D_{i}=\left\{u_{i}^{\prime}=0\right\}$ for $1 \leq i \leq m$. We can write $u_{i}=u_{i}^{\prime} g_{i}$ for $1 \leq i \leq m$, where $g_{i} \in \mathcal{O}_{z}$ and $g_{i}(z) \neq 0$. It is easy to see that if $\varepsilon>0$ is small enough, then there exists $\varepsilon^{\prime}>0$ such that

$$
D_{u^{\prime}}\left(\log \varepsilon^{\prime}+s \alpha\right) \subseteq D_{u}(\log \varepsilon+s \alpha)
$$

for all $s \leq 0$. This gives $h_{u^{\prime}}(\alpha)=h_{u^{\prime}, \varepsilon^{\prime}}(\alpha) \geq h_{u, \varepsilon}(\alpha)=h_{u}(\alpha)$ and the reverse inequality follows by symmetry. Thus (iii) holds. 
Finally we prove (iv). Thus pick a point $z \in Z$, and a set of local coordinates $u$ at $z$. Pick $0<\varepsilon \ll 1$ and $z^{\prime} \in D_{u}(\log \varepsilon) \cap Z$. Then $u^{\prime}:=u-u\left(z^{\prime}\right)$ defines local coordinates at $z^{\prime}$ and for any $\alpha$ as above and any $s \leq 0$ we have

$$
D_{u^{\prime}}(\log \varepsilon+s \alpha) \subseteq D_{u}(\log 2 \varepsilon+s \alpha) \quad \text { and } \quad D_{u}(\log \varepsilon+s \alpha) \subseteq D_{u^{\prime}}(\log 2 \varepsilon+s \alpha) .
$$

This implies that $h_{z^{\prime}}=h_{z^{\prime}, u^{\prime}, \varepsilon} \geq h_{z, u, 2 \varepsilon}=h_{z}$ and, similarly, $h_{z} \geq h_{z^{\prime}}$. Thus $z \mapsto$ $h_{z}$ is locally constant on $Z$, which completes the proof since $Z$ is connected.

Now assume $\alpha_{i}=0$ for $i>m$ and $\alpha_{i}>0$ for $1 \leq i \leq m$. The number

$$
\tau_{Z, D, \alpha}(\varphi):=h_{\varphi, Z, D}(\alpha)
$$

is called the Kiselman number ${ }^{6}$ of $\varphi$ along $Z$ with weight $\alpha_{i}$ along $D_{i}$. As explained in Lemma 3.1, it does not depend on the choice of coordinates $u_{i}$ defining the hypersurfaces $D_{i}$. However, given such coordinates, it follows from the convexity of $H$ that we have the estimate

$$
\varphi \leq \tau_{Z, D, \alpha}(\varphi) \max _{i \leq m} \frac{1}{\alpha_{i}} \log \left|u_{i}\right|+O(1),
$$

near $z$. From this inequality we easily deduce

Lemma 3.2. Suppose $\varphi, \psi$ are psh functions defined near some Zariski general point $z \in Z$. Write $\tau=\tau_{Z, D, \alpha}$. Then:

(i) if $\varphi \leq \psi+O(1)$ near $z$, then $\tau(\varphi) \geq \tau(\psi)$;

(ii) $\tau(\max \{\varphi, \psi\})=\min \{\tau(\varphi), \tau(\varphi)\}$.

Proof. The inequality in (i) follows immediately from the definition. As for (ii), note that (i) implies $\tau(\max \{\varphi, \psi\}) \leq \min \{\tau(\varphi), \tau(\varphi)\}$. The reverse inequality follows from (3.4).

Remark 3.3. It is also true that $\tau(\varphi+\psi)=\tau(\varphi)+\tau(\psi)$, but we do not need this fact.

Remark 3.4. Using the same construction, we can define $\tau_{Z, D, \alpha}$ when $Z$ and the $D_{i}$ are germs of complex submanifolds at a point in a complex manifold.

Remark 3.5. When $\alpha_{i}=1$ for $1 \leq i \leq m$, the choice of hypersurfaces $D_{i}$ play no role; in this case the Kiselman number is equal to the Lelong number along $Z$ [Lel69].

3.3. Kiselman numbers and quasimonomial valuations. Let $U$ be a complex manifold, $x \in U$ a point and $V$ the germ at $x$ of a complex submanifold of $U$. We allow for the case $V=x$ but assume that $V$ has codimension $\geq 1$. As in $\$ 2.2 .1$ let $\mathcal{O}_{x, V}$ be the localization of $\mathcal{O}_{x}$ at the ideal $I_{V} \cdot \mathcal{O}_{x}$ and let $\mathfrak{m}_{x, V}$ be the maximal ideal of $\mathcal{O}_{x, V}$. Let $v$ be a quasimonomial valuation of $\mathcal{O}_{x, V}$. We want to associate to $v$ a Kiselman number on a suitable modification.

Consider a projective birational morphism $\pi: X \rightarrow \operatorname{Spec} \mathcal{O}_{x, V}$ that is adapted to $v$ in the sense of 2.1 .1 . Thus there exist prime divisors $D_{1}, \ldots, D_{m}$ on $X$ such that $\sum D_{i}$ has simple normal crossing singularities, and an irreducible component

\footnotetext{
${ }^{6}$ In [Kis87, Kis94, the Kiselman number is called a refined Lelong number whereas Demailly Dem87. calls it a directional Lelong number.
} 
$Z$ of $\bigcap_{i=1}^{m} D_{i}$ such that $v$ is monomial with weight $\alpha_{i}>0$ on $D_{i}$ for $1 \leq i \leq m$. The assumption that $v \geq 0$ on $\mathcal{O}_{x, V}$ implies that $\pi(Z) \subset V$. Let $\xi$ be the generic point of $Z$ and pick functions $u_{i} \in \mathcal{O}_{X, \xi}, 1 \leq i \leq m$, such that $D_{i}=\left(u_{i}=0\right)$. Thus the functions $u_{i}$ are regular on a Zariski open subset of $Z$.

Using the construction and conventions of \$2.2.1, after shrinking $U$ a little, the projective birational morphism $\pi: X \rightarrow \operatorname{Spec}_{x, V}$ gives rise to a complex manifold $X^{\text {an }}$ and a proper modification $\pi^{\text {an }}: X^{\text {an }} \rightarrow U \backslash W$, where $W \subseteq U$ is a complex subvariety not containing $V$.

Further, after again shrinking $U$ and increasing $W$ if necessary, there exists an open subset $\Omega$ of $X^{\text {an }}$ on which the functions $u_{i}, 1 \leq i \leq m$, are holomorphic and such that the following properties hold: the sets $D_{i}^{\text {an }}:=\left(u_{i}=0\right)$ are complex submanifolds of $\Omega$ of codimension one, meeting transversely along the connected submanifold $Z^{\text {an }}:=\bigcap_{1<i<m} D_{i}^{\text {an }}$. Further $\pi^{\text {an }}\left(Z^{\text {an }}\right) \supseteq V \backslash W$.

Let $\tau=\tau_{Z^{\text {an }}, D^{\text {an }}, \alpha}$ denote the Kiselman number with respect to the data above, see $\$ 3.2$.

Definition 3.6. If $\varphi$ is the germ of a psh function at $x$, then we define

$$
v(\varphi):=\tau\left(\varphi \circ \pi^{\mathrm{an}}\right) .
$$

Note that this definition a priori depends on a lot of choices made above. However, we have:

Proposition 3.7. The definition of $v(\varphi)$ does not depend on any choices made as long as the birational morphism $\pi: X \rightarrow \operatorname{Spec} \mathcal{O}_{x, V}$ is adapted to $v$.

We shall prove this result in $\$ 3.4$ using multiplier ideals, see Remark 3.13. For now, we only treat the following special case.

Lemma 3.8. If $\mathfrak{b} \subseteq \mathcal{O}_{x}$ is a nonzero ideal, then

$$
v\left(\mathfrak{b} \cdot \mathcal{O}_{x, V}\right)=\tau\left((\log |\mathfrak{b}|) \circ \pi^{\text {an }}\right) .
$$

Proof. Note that both sides of $(3.6)$ depend continuously on the weight $\alpha \in$ $\mathbf{R}_{>0}^{m}$. Hence we may assume that the $\alpha_{i}$ are rationally independent. In view of Lemma 3.2 we may also assume that $\mathfrak{b}$ is generated by a single element $f \in \mathcal{O}_{x}$. We must prove that $\tau\left(\log |f| \circ \pi^{\text {an }}\right)=v(f)$.

Consider a Zariski general closed point $z \in Z$ and pick functions $u_{m+1}, \ldots, u_{n} \in$ $\mathcal{O}_{X, z}$ such that $u:=\left(u_{1}, \ldots, u_{n}\right)$ define local algebraic coordinates on $X$ at $z$. Write $u^{\prime}:=\left(u_{1}, \ldots, u_{m}\right)$ and $u^{\prime \prime}:=\left(u_{m+1}, \ldots, u_{n}\right)$. Consider the expansion of $f \circ \pi$ as a formal power series in $\widehat{\mathcal{O}_{X, \xi}} \simeq \mathbf{C} \llbracket u \rrbracket=\mathbf{C} \llbracket u^{\prime \prime} \rrbracket \llbracket u^{\prime} \rrbracket$ :

$$
f \circ \pi=\sum_{\beta \in \mathbf{Z}_{\geq 0}^{m}, \gamma \in \mathbf{Z}_{\geq 0}^{n-m}} a_{\beta \gamma}\left(u^{\prime}\right)^{\beta}\left(u^{\prime \prime}\right)^{\gamma}=\sum_{\beta \in \mathbf{Z}_{\geq 0}^{m}} a_{\beta}\left(u^{\prime \prime}\right)\left(u^{\prime}\right)^{\beta},
$$

where $a_{\beta, \gamma} \in \mathbf{C}$ and

$$
a_{\beta}\left(u^{\prime \prime}\right)=\sum_{\gamma \in \mathbf{Z}_{\geq 0}^{n-m}} a_{\beta \gamma}\left(u^{\prime \prime}\right)^{\gamma} \in \mathbf{C} \llbracket u^{\prime \prime} \rrbracket \subseteq \mathbf{C} \llbracket u \rrbracket .
$$


Since the $\alpha_{i}$ are rationally independent, there exists a unique $\bar{\beta}$ minimizing $\beta \cdot \alpha:=$ $\beta_{1} \alpha_{1}+\cdots+\beta_{m} \alpha_{m}$ over all $\beta$ for which $a_{\beta} \not \equiv 0$. By definition, we then have

$$
v(f)=\bar{\beta} \cdot \alpha .
$$

Since the point $z \in Z$ was generically chosen, it corresponds to a point, also denoted $z$, on the complex manifold $Z^{\text {an }}$. We may assume that such that $f$ is holomorphic near $\pi^{\text {an }}(z) \in U \backslash W$. Pick $0<\varepsilon \ll 1$ such that $f \circ \pi^{\text {an }}$ is holomorphic on the open polydisk $\left|u_{i}\right|<\varepsilon, 1 \leq i \leq n$. The first series in (3.7) is then the Taylor series of the holomorphic function $f \circ \pi^{\text {an }}$ at $z$ in the analytic coordinates $u$ and this series converges locally uniformly in the polydisk $\|u\|<\varepsilon$. Further, for every $\beta$, the series in (3.8) converges locally uniformly for $\left\|u^{\prime \prime}\right\|<\varepsilon$ to a holomorphic function $a_{\beta}\left(u^{\prime \prime}\right)$ and the second series in (3.7) converges locally uniformly for $\|u\|<\varepsilon$.

By assumption, the holomorphic function $a_{\bar{\beta}}$ is not constantly equal to zero. After moving $z$ (but keeping $z \in Z^{\text {an }}$ ) a little and translating the coordinates $u_{m+1}, \ldots, u_{n}$ accordingly, we may assume that $a_{\bar{\beta}}(0) \neq 0$. Let us use the notation of $\oint 3.2$. For $0<\varepsilon \ll 1$ we have

$$
\log |f| \circ \pi^{\text {an }}=\log \left|f \circ \pi^{\text {an }}\right| \sim \log \left|a_{\bar{\beta}}(0)\left(u^{\prime}\right)^{\bar{\beta}}\right| \sim s \bar{\beta} \cdot \alpha
$$

on the set $S_{u}(\log \varepsilon+s \alpha)$, as $s \rightarrow-\infty$. This implies that

$$
\tau\left(\log |f| \circ \pi^{\text {an }}\right)=\bar{\beta} \cdot \alpha=v(f)
$$

as was to be shown.

3.4. Multiplier ideal sheaves and Demailly approximation. To a psh function $\varphi$ on a complex manifold $U$ is associated a multiplier ideal sheaf $\mathcal{J}(\varphi)$. This is an ideal sheaf on $U$ whose stalk at a point $x$ is the set of holomorphic germs $f \in \mathcal{O}_{x}$ such that $|f|^{2} e^{-2 \varphi}$ is locally integrable at $x$. The coherence of $\mathcal{J}(\varphi)$ is a nontrivial result due to Nadel [Nad89, Nad90, which can be proved using Hörmander's $L^{2}$-estimates, see [DK01, Thm 4.1].

Recall from $\$ 3.1$ the definition of the jumping number $c_{x}^{\mathfrak{q}}(\varphi)$ of $\varphi$ at $x$ relative to an ideal $\mathfrak{q}$ on $U$. Given $\mu>0$ consider the colon ideal $a_{\mu}=(\mathcal{J}(\mu \varphi): \mathfrak{q})$ on $U$. This is an ideal sheaf on $U$ whose stalk at a point $x \in U$ is given by

$$
\mathfrak{a}_{\mu} \cdot \mathcal{O}_{x}:=\left\{\left.h \in \mathcal{O}_{x}|| h\right|^{2}|\mathfrak{q}|^{2} e^{-2 \mu \varphi} \text { is locally integrable at } x\right\} .
$$

Since $\mathcal{J}(\mu \varphi)$ and $\mathfrak{q}$ are coherent, so is $a_{\mu}$.

Lemma 3.9. We have $c_{x}^{\mathfrak{q}}(\varphi)<\mu$ iff $\mathfrak{a}_{\mu} \cdot \mathcal{O}_{x} \neq \mathcal{O}_{x}$. As a consequence, the function $x \mapsto c_{x}^{\mathfrak{q}}$ is lower semicontinuous in the analytic Zariski topology on $U$.

Proof. The first statement is clear. Hence, for $\mu>0$, the set

$$
V_{\mu}^{-}:=\left\{x \in U \mid c_{x}^{\mathfrak{q}}(\varphi)<\mu\right\}
$$

is equal to the support of the coherent sheaf $\mathcal{O}_{U} / \mathfrak{a}_{\mu}$ and in particular an analytic subset of $U$. It follows that for $\lambda>0$, the set

$$
V_{\lambda}:=\left\{x \in U \mid c_{x}^{\mathfrak{q}}(\varphi) \leq \lambda\right\}=\bigcap_{\mu>\lambda} V_{\mu}^{-}
$$

is also an analytic subset of $U$. This concludes the proof. 
Remark 3.10. Conjecture A' in $\$ 3.1$ is equivalent to a semicontinuity statement about multiplier ideals. Indeed, define $\mathcal{J}^{+}(\varphi)$ as the increasing (locally stationary) limit of $\mathcal{J}((1+\varepsilon) \varphi)$ as $\varepsilon \searrow 0$. Then Conjecture A' precisely says that $\mathcal{J}^{+}(\varphi)=\mathcal{J}(\varphi)$.

If $f_{1}, \ldots, f_{m}$ are holomorphic functions on $U$, generating an ideal sheaf $\mathfrak{q}$ and if $\log |\mathfrak{q}|$ is the corresponding psh function on $U$ defined by (3.1), then we have

$$
\mathcal{J}(\log |\mathfrak{q}|)=\mathcal{J}(\mathfrak{q}),
$$

where the right-hand side is defined as in 2.1 , see [DK01, Proposition 1.7].

Lemma 3.11. If $\varphi \geq p \log |\mathfrak{q}|+O(1)$ for some integer $p \geq 1$, then $\mathcal{J}(\varphi) \supseteq \mathfrak{q}^{p}$.

Proof. In view of the assumption and (3.12) we have

$$
\mathcal{J}(\varphi) \supseteq \mathcal{J}(p \log |\mathfrak{q}|)=\mathcal{J}\left(\mathfrak{q}^{p}\right) \supseteq \mathfrak{q}^{p} .
$$

Here the last inclusion holds since $\mathcal{J}(\mathfrak{a}) \supseteq \mathfrak{a}$ for any ideal $\mathfrak{a}$.

Now fix a psh function $\varphi$ on $U$. For $j \geq 1$ set

$$
\mathfrak{b}_{j}:=\mathcal{J}(j \varphi) \text {. }
$$

It follows from [DEL00] that $\mathfrak{b}_{\bullet}=\left(\mathfrak{b}_{j}\right)_{j=1}^{\infty}$ is a subadditive sequence of ideals on $U$. The following result (which was known in the case $V=x$, see DK01, Theorem 4.2] and [BFJ08, Theorem 5.5]) allows us to understand the singularities of $\varphi$ in terms of those of $\mathfrak{b}_{\bullet}$.

Proposition 3.12. Let $x$ be any point in $U$ and let $V$ be the germ at $x$ of a proper complex submanifold. Define $\mathcal{O}_{x, V}$ as in $\$ 2.2$. Then the following properties hold:

(i) for every nonzero ideal $\mathfrak{q} \subseteq \mathcal{O}_{x}$ we have $c_{x}^{\mathfrak{q}}(\varphi)=\operatorname{lct}_{x}^{\mathfrak{q}}\left(\mathfrak{b}_{\bullet}\right)$;

(ii) the subadditive sequence $\mathfrak{b}_{\bullet} \cdot \mathcal{O}_{x, V}$ has controlled growth;

(iii) for every quasimonomial valuation $v$ on $\mathcal{O}_{x, V}$ we have $v(\varphi)=v\left(\mathfrak{b}_{\bullet} \cdot \mathcal{O}_{x, V}\right)$.

Remark 3.13. In (iii) we compute $v(\varphi)$ as a Kiselman number of the pullback of $\varphi$ under a suitable proper modification, the latter being the analytification of a blowup of $\operatorname{Spec} \mathcal{O}_{x, V}$, see $\$ 3.3$. Since the quantity $v\left(\mathfrak{b}_{\bullet} \cdot \mathcal{O}_{x, V}\right)$ does not depend on any choices made, we see that $v(\varphi)$ is uniquely defined. Thus we obtain a proof of Proposition 3.7.

The proof of Proposition 3.12 relies on a fundamental approximation procedure due to Demailly [Dem92, Dem93]. We refer to [DK01, §4] for details on what follows.

Let $\varphi$ be a psh function defined in some pseudoconvex domain $B \subseteq U$ containing $x$. For $p \geq 1$ consider the Hilbert space

$$
\mathcal{H}_{p}:=\left\{\left.f \in \mathcal{O}(B)\left|\int_{B}\right| f\right|^{2} e^{-2 p \varphi}<\infty\right\},
$$

with the natural inner product. It is a fact that for every $y \in B$, the elements of $\mathcal{H}_{p}$ generate the stalk at $y$ of the multiplier ideal sheaf $\mathfrak{b}_{p}:=\mathcal{J}(p \varphi)$. Define

$$
\varphi_{p}:=\frac{1}{p} \sup \left\{\left.\log |f|\left|\int_{B}\right| f\right|^{2} e^{-2 p \varphi} \leq 1\right\} .
$$


Then $\varphi_{p}$ is psh on $B$. It follows from the Ohsawa-Takegoshi Theorem that

$$
\varphi \leq \varphi_{p}+\frac{C}{p}
$$

on $B$, for some constant $C$ not depending on $\varphi$ or $p$. For any $y \in B$ and any nonzero ideal $\mathfrak{q} \subseteq \mathcal{O}_{y}$ we also have

$$
\begin{aligned}
\left(p \operatorname{lct}_{y}^{\mathfrak{q}}\left(\mathfrak{b}_{p}\right)\right)^{-1}=c_{y}^{\mathfrak{q}}\left(\varphi_{p}\right)^{-1} & \leq c_{y}^{\mathfrak{q}}(\varphi)^{-1} \\
& \leq c_{y}^{\mathfrak{q}}\left(\varphi_{p}\right)^{-1}+\frac{1}{p}=\left(p \operatorname{lct}_{y}^{\mathfrak{q}}\left(\mathfrak{b}_{p}\right)\right)^{-1}+\frac{1}{p} .
\end{aligned}
$$

Here the two equalities follow from 3.12 whereas the first inequality results from (3.13). The second inequality is proved in [DK01, Thm 4.2 (3)] in the case $\mathfrak{q}=\mathcal{O}_{U}$ and the same proof works in the general case.

Proof of Proposition 3.12. Clearly (i) follows from (3.14) with $y=x$ by letting $p \rightarrow \infty$. It remains to prove (ii) and (iii).

We use the notation of $\$ 3.3$. Write $\tau=\tau_{Z, D, \alpha}$. It follows from (3.13) and from Proposition 3.7 that

$$
\tau\left(\varphi \circ \pi^{\mathrm{an}}\right) \geq \tau\left(\varphi_{p} \circ \pi^{\mathrm{an}}\right)=\frac{1}{p} v\left(\mathfrak{b}_{p} \cdot \mathcal{O}_{x, V}\right)
$$

for any $p \geq 1$.

We will show that if $f \in \mathcal{H}_{p}$, then

$$
v(f)+A(v) \geq p \tau\left(\varphi \circ \pi^{\mathrm{an}}\right) .
$$

Grant (3.16) for the moment. We then have

$$
\frac{1}{p} v\left(\mathfrak{b}_{p} \cdot \mathcal{O}_{x, V}\right) \leq \tau\left(\varphi \circ \pi^{\mathrm{an}}\right) \leq \frac{1}{p} v\left(\mathfrak{b}_{p} \cdot \mathcal{O}_{x, V}\right)+\frac{1}{p} A(v) .
$$

Letting $p$ tend to infinity we see that $\tau\left(\varphi \circ \pi^{\text {an }}\right)=v\left(\mathfrak{b}_{\bullet} \cdot \mathcal{O}_{x, V}\right)$, proving (iii). In particular, $v(\varphi)=\tau\left(\varphi \circ \pi^{\text {an }}\right)$ is well defined independently of any choices made so we have established Proposition 3.7. Since $v$ was an arbitrary quasimonomial valuation on $\mathcal{O}_{x, V}$ we also see that $\mathfrak{b}_{\bullet} \cdot \mathcal{O}_{x, V}$ has controlled growth, proving (ii).

It only remains to prove (3.16). Since both sides of (3.16) depend continuously on the weight $\alpha$, we may assume that $\alpha_{1}, \ldots, \alpha_{m}$ are rationally independent. We now argue as in the proof of Lemma 3.8, recycling the notation from that proof. Thus we have the expansion (3.7) and we have $a_{\bar{\beta}}(0) \neq 0$ for the unique $\bar{\beta} \in \mathbf{Z}_{\geq 0}^{m}$ for which $\bar{\beta} \cdot \alpha=v(f)$.

Now fix $K \gg 1$. Define a sequence of disjoint open subsets $\left(\Omega_{k}\right)_{k \geq 0}$ of $\Omega$ by

$$
\Omega_{k}:=\bigcap_{i=1}^{m}\left\{-(K+k)<\frac{\log \left|u_{i}\right|}{\alpha_{i}}<1-(K+k)\right\} \cap \bigcap_{i=m+1}^{n}\left\{-K<\log \left|u_{i}\right|<1-K\right\} .
$$

For large $k$ we then have the following estimates on $\Omega_{k}$ :

$$
\log \left|f \circ \pi^{\mathrm{an}}\right| \geq-k v(f)+O(1) \quad \text { and } \quad \varphi \circ \pi^{\mathrm{an}} \leq-k \tau\left(\varphi \circ \pi^{\mathrm{an}}\right)+O(1) .
$$


Here the second estimate follows from (3.4). Let $\eta$ be a nonvanishing holomorphic volume form near $x$ and write $\left(\pi^{\text {an }}\right)^{*} \eta=J \pi^{\text {an }} \cdot \eta_{u}$ near $z$, where $\eta_{u}:=d u_{1} \wedge \cdots \wedge$ $d u_{n}$. We then have

$$
\log \left|J \pi^{\mathrm{an}}\right|=\sum_{i=1}^{m}\left(A_{i}-1\right) \log \left|u_{i}\right|+O(1)
$$

near $z$, where $A_{i} \in \mathbf{Z}_{>0}$. Further, we have

$$
A(v)=\sum_{i=1}^{m} \alpha_{i} A_{i}
$$

As $k \rightarrow \infty$, we then have

$$
\log \left|J \pi^{\mathrm{an}}\right| \sim-k\left(A(v)-\sum_{i=1}^{m} \alpha_{i}\right)+O(1)
$$

on $\Omega_{k}$. Moreover, the volume of $\Omega_{k}$ can be estimated by

$$
\log \int_{\Omega_{k}}(\sqrt{-1})^{n} \eta_{u} \wedge \overline{\eta_{u}}=-2 k \sum_{i=1}^{m} \alpha_{i}+O(1)
$$

as $k \rightarrow \infty$.

Note that if $K$ is large enough, then $\pi^{\text {an }}$ is biholomorphic on $\Omega_{k}$ and $\pi^{\text {an }}\left(\Omega_{k}\right)$ is contained in $B$ for all $k \geq 0$. Thus we get

$$
\begin{gathered}
+\infty>\int_{B}|f|^{2} e^{-2 p \varphi}(\sqrt{-1})^{n} \eta \wedge \bar{\eta} \geq \sum_{k=0}^{\infty} \int_{\pi^{\mathrm{an}}\left(\Omega_{k}\right)}|f|^{2} e^{-2 p \varphi}(\sqrt{-1})^{n} \eta \wedge \bar{\eta} \\
=\sum_{k=0}^{\infty} \int_{\Omega_{k}}\left|f \circ \pi^{\mathrm{an}}\right|^{2} e^{-2 p \varphi \circ \pi^{\mathrm{an}}}\left|J \pi^{\mathrm{an}}\right|^{2}(\sqrt{-1})^{n} \eta_{u} \wedge \overline{\eta_{u}} \\
\gtrsim \sum_{k=0}^{\infty} \exp \left(-2 k v(f)+2 k p \tau\left(\varphi \circ \pi^{\mathrm{an}}\right)-k\left(A(v)-\sum_{i=1}^{m} \alpha_{i}\right)\right) \int_{\Omega_{k}}(\sqrt{-1})^{n} \eta_{u} \wedge \overline{\eta_{u}} \\
\gtrsim \sum_{k=0}^{\infty} \exp \left(-2 k\left(v(f)-p \tau\left(\varphi \circ \pi^{\mathrm{an}}\right)+A(v)\right)\right),
\end{gathered}
$$

which yields (3.16) (with strict inequality).

\section{Proof of the main Results}

We are now ready to prove Theorem $\mathrm{D}$ from the introduction and its variant Theorem D' from \$3.1. Consider a germ of a psh function $\varphi$ at a point $x$ in a complex manifold of dimension $n$ and let $\mathfrak{q} \subseteq \mathcal{O}_{x}$ be a nonzero ideal such that $c_{x}^{\mathfrak{q}}(\varphi)<\infty$. Let $U$ be a small open neighborhood of $x$ such that $\varphi$ and $\mathfrak{q}$ are defined on an open neighborhood of $\bar{U}$. Also fix a nonvanishing holomorphic volume form $\eta$ in a neighborhood of $\bar{U}$ and compute all volumes with respect to the positive measure $(\sqrt{-1})^{n} \eta \wedge \bar{\eta}$. 
4.1. Analytic reduction. As in $\S 3.4$ set

$$
V_{\mu}:=\left\{y \in U \mid c_{y}^{\mathfrak{q}}(\varphi) \leq \mu\right\}
$$

for $\mu \geq \lambda:=c_{x}^{\mathfrak{q}}(\varphi)$ and

$$
V:=V_{\lambda}=\left\{y \in U \mid c_{y}^{\mathfrak{q}}(\varphi) \leq \lambda\right\} .
$$

Note that $x \in V$. By the lower semicontinuity of $y \mapsto c_{y}^{\mathfrak{q}}$ (see Lemma 3.9, $V_{\mu}$ is a proper analytic subset of $U$ for any $\mu \geq \lambda$ and $V$ is the decreasing intersection of $V_{\mu}$ for all $\mu>\lambda$. Using the fact that $\varphi$ and $\mathfrak{q}$ are defined in a neighborhood of $\bar{U}$ we deduce the existence of $\mu>\lambda$ such that $V=V_{\mu}$.

Lemma 4.1. In order to prove Theorem $D$ ', it suffices to assume that $V$ is smooth at $x$ and that $\varphi \geq p \log \left|I_{V}\right|+O(1)$ near $x$ for some integer $p \geq 0$.

Proof. We can replace $x$ by a Zariski general point in $V$. Indeed, we have $c_{y}^{\mathfrak{q}}(\varphi)=$ $\lambda$ for a Zariski general point $y \in V$, and if the estimate

$$
\operatorname{Vol}\left\{y^{\prime} \in U_{y}\left|\lambda \varphi\left(y^{\prime}\right)-\log \right| \mathfrak{q} \mid<\log r\right\} \gtrsim r^{2}
$$

holds for every neighborhood $U_{y}$ of any point $y$ in a dense subset of $V$, then it also holds for every neighborhood of $x$. In particular, we may assume that $V$ is smooth at $x$.

Pick generators of $I_{V} \cdot \mathcal{O}_{x}$. After shrinking $U$, we may assume these generators are defined on $U$ and that the associated psh function $\log \left|I_{V}\right|$, defined as in (3.1), is negative on $U$. For an integer $p>0$ define

$$
\tilde{\varphi}:=\max \left\{\varphi, p \log \left|I_{V}\right|\right\} .
$$

We claim that $c_{x}^{\mathfrak{q}}(\tilde{\varphi})=c_{x}^{\mathfrak{q}}(\varphi)$ for $p \gg 0$. This will allow us to replace $\varphi$ by $\tilde{\varphi}$ and complete the proof. Indeed, we have $\varphi \leq \tilde{\varphi}$ so if the estimate $(3.2)$ holds with $\varphi$ replaced by $\tilde{\varphi}$, then it must also hold for $\varphi$.

To prove the claim, pick $\mu>\lambda$ such that $V_{\mu}=V$. Consider the colon ideal $a_{\mu}=(\mathcal{J}(\mu \varphi): \mathfrak{q})$ on $U$. This is a coherent ideal sheaf on $U$ whose stalk at $y \in U$ is given by

$$
\mathfrak{a}_{\mu} \cdot \mathcal{O}_{y}:=\left\{\left.h \in \mathcal{O}_{y}|| h\right|^{2}|\mathfrak{q}|^{2} e^{-2 \mu \varphi} \text { is locally integrable at } y\right\} .
$$

The fact that $V_{\mu}=V$ implies that the zero locus of $\mathfrak{a}_{\mu}$ is equal to $V$. Hence the Nullstellensatz implies that there exists $N \geq 1$ such that $I_{V}^{N} \subseteq \mathfrak{a}_{\mu}$. Now pick the integer $p>0$ large enough so that

$$
p>\frac{N}{\mu-\lambda} .
$$

Pick any $\lambda^{\prime} \in(\lambda, \mu)$ such that $p>N /\left(\mu-\lambda^{\prime}\right)$. For $0<r \ll 1$ define Borel subsets $U_{r}, \tilde{U}_{r}$ and $U_{r}^{\prime}$ of $U$ by

$$
\begin{aligned}
U_{r} & :=\left\{\lambda^{\prime} \varphi-\log |\mathfrak{q}|<\log r\right\} \\
\tilde{U}_{r} & :=\left\{\lambda^{\prime} \tilde{\varphi}-\log |\mathfrak{q}|<\log r\right\} \\
U_{r}^{\prime} & :=\left\{\mu \varphi-\log |\mathfrak{q}|-N \log \left|I_{V}\right|<\log r\right\} .
\end{aligned}
$$


It follows from the choice of $p$ that $U_{r} \subseteq \tilde{U}_{r} \cup U_{r}^{\prime}$. The inclusion $I_{V}^{N} \subseteq \mathfrak{a}_{\mu}$ guarantees that, after possibly shrinking $U$, we have

$$
\int_{0}^{\infty} \operatorname{Vol}\left(U_{r}^{\prime}\right) \frac{d r}{r^{3}}<\infty
$$

Indeed, if we set $F:=\exp \left(N \log \left|I_{V}\right|+\log |\mathfrak{q}|-\mu \varphi\right)$, then, after shrinking $U$,

$$
\infty>\int_{U} F^{2}=2 \int_{0}^{\infty} \operatorname{Vol}(U \cap\{F>t\}) t d t=2 \int_{0}^{\infty} \operatorname{Vol}\left(U_{r}^{\prime}\right) \frac{d r}{r^{3}},
$$

where the last equality follows from setting $t=1 / r$.

On the other hand, the fact that $\lambda^{\prime}>\lambda=c_{x}^{\mathfrak{q}}(\varphi)$ implies that

$$
\int_{0}^{\infty} \operatorname{Vol}\left(U_{r}\right) \frac{d r}{r^{3}}=\infty
$$

The inclusion $U_{r} \subseteq \tilde{U}_{r} \cup U_{r}^{\prime}$ then gives

$$
\int_{0}^{\infty} \operatorname{Vol}\left(\tilde{U}_{r}\right) \frac{d r}{r^{3}}=\infty
$$

so that $c_{x}^{\mathfrak{q}}(\tilde{\varphi}) \leq \lambda^{\prime}$. Letting $\lambda^{\prime} \rightarrow \lambda$ we get $c_{x}^{\mathfrak{q}}(\tilde{\varphi}) \leq \lambda$. But $\tilde{\varphi} \geq \varphi$, so we must have $c_{x}^{\mathfrak{q}}(\tilde{\varphi}) \geq c_{x}^{\mathfrak{q}}(\varphi)=\lambda$ and hence $c_{x}^{\mathfrak{q}}(\tilde{\varphi})=c_{x}^{\mathfrak{q}}(\varphi)$, establishing the claim and completing the proof of the lemma.

Remark 4.2. The proof of Lemma 4.1 can be viewed as an analytic analogue of the arguments in [JM12, $§ 7.4]$.

4.2. End of proof. Let $x$ and $V$ be as above. In particular, $V$ is smooth at $x$. Let $\mathcal{O}_{x, V}$ be the localization of $\mathcal{O}_{x}$ at the ideal $I_{V} \cdot \mathcal{O}_{x}$. Then $\mathcal{O}_{x, V}$ is a regular local ring with maximal ideal $\mathfrak{m}_{x, V}=I_{V} \cdot \mathcal{O}_{x, V}$. Its dimension is equal to the codimension of $V$ and hence bounded by $n$. It is also an excellent ring. Indeed, $\mathcal{O}_{x}$ is isomorphic to the ring of convergent power series in $n$ variables, hence excellent, see [Mat80, Theorem 102], and excellence is preserved by localization.

Set $\mathfrak{b}_{j}=\mathcal{J}(j \varphi)$ for $j \geq 0$. Then $\mathfrak{b}_{\bullet} \cdot \mathcal{O}_{x, V}$ is a subadditive system of ideals having controlled growth, see Proposition 3.12. By Lemma 4.1 we may assume $\varphi \geq p \log \left|I_{V}\right|+O(1)$; hence Lemma 3.11 implies $\mathfrak{b}_{j} \cdot \mathcal{O}_{x, V} \supseteq \mathfrak{m}_{x, V}^{p j}$ for all $j \geq 1$.

From the definition of $V=V_{\lambda}$ and from Proposition 3.12 we see that

$$
\operatorname{lct}_{y}^{\mathfrak{q}}\left(\mathfrak{b}_{\bullet}\right)=c_{y}^{\mathfrak{q}}(\varphi)=\lambda
$$

for every $y \in V$. Lemma 2.7 then shows that

$$
\operatorname{lct}^{\mathfrak{q} \cdot \mathcal{O}_{x, V}}\left(\mathfrak{b}_{\bullet} \cdot \mathcal{O}_{x, V}\right)=\lambda \text {. }
$$

Recall that we assume that Conjecture C' holds in rings of dimension at most $n$. Proposition 2.3 implies that Conjecture E' also holds in rings of dimension at most $n$. We can thus find a quasimonomial valuation $v$ on $\mathcal{O}_{x, V}$ such that

$$
\frac{A(v)+v\left(\mathfrak{q} \cdot \mathcal{O}_{x, V}\right)}{v\left(\mathfrak{b}_{\bullet} \cdot \mathcal{O}_{x, V}\right)}=\lambda .
$$

Consider a projective birational morphism $\pi: X \rightarrow \operatorname{Spec} \mathcal{O}_{x, V}$ such that $\pi$ defines a $\log$ resolution of $\mathfrak{q}$ and such that $X$ is adapted to $v$. Thus $v$ is given by data $Z, D, \alpha$ as in 2.1 .1 . 
We analytify $\pi$ following $\$ 2.2 .1$ and $\$ 3.3$. Let $\tau$ denote the Kiselman number with respect to the data $Z^{\text {an }}, D^{\text {an }}, \alpha$, see $\$ 3.2$. We know from Proposition 3.12 (iii) and Remark 3.13 that

$$
\tau\left(\varphi \circ \pi^{\mathrm{an}}\right)=v\left(\mathfrak{b}_{\bullet} \cdot \mathcal{O}_{x, V}\right)
$$

Thus (4.3) yields

$$
\lambda \tau\left(\varphi \circ \pi^{\mathrm{an}}\right)=A(v)+v(\mathfrak{q}) .
$$

We use the notation from $\$ 3.3$. Pick a Zariski general point $z \in Z^{\text {an }}$. Then

$$
\log |\mathfrak{q}| \circ \pi^{\text {an }}=\sum_{i=1}^{m} c_{i} \log \left|u_{i}\right|+O(1)
$$

near $z$, where $c_{i}=\operatorname{ord}_{D_{i}}(\mathfrak{q}) \geq 0$; see the end of 2.2 .1 . We also have

$$
\log \left|J \pi^{\mathrm{an}}\right|=\sum_{i=1}^{m}\left(A_{i}-1\right) \log \left|u_{i}\right|+O(1),
$$

where $A_{i} \in \mathbf{Z}_{>0}$, see 3.19 . Finally, recall from 3.4 that

$$
\varphi \circ \pi^{\mathrm{an}} \leq \tau\left(\varphi \circ \pi^{\mathrm{an}}\right) \max _{1 \leq i \leq m} \frac{1}{\alpha_{i}} \log \left|u_{i}\right|+O(1) .
$$

Fix $K \gg 1$ and define disjoint open subsets $\Omega_{k}, k \geq 0$, of $\Omega$ as in (3.18). As $k \rightarrow \infty$, we then have the following estimates on $\Omega_{k}$ :

$$
\begin{gathered}
\varphi \circ \pi^{\mathrm{an}} \leq-k \tau\left(\varphi \circ \pi^{\mathrm{an}}\right)+O(1), \\
\log |\mathfrak{q}| \circ \pi^{\mathrm{an}} \geq-k \sum_{i=1}^{m} c_{i} \alpha_{i}+O(1)=-k v(\mathfrak{q})+O(1) .
\end{gathered}
$$

Using (4.5) these estimates imply that

$$
\lambda \varphi-\log |\mathfrak{q}| \leq-k\left(\lambda \tau\left(\varphi \circ \pi^{\text {an }}\right)-v(\mathfrak{q})\right)+O(1)=-k A(v)+O(1)
$$

on the open set $\pi^{\text {an }}\left(\Omega_{k}\right) \subset U$. For $1 \leq i \leq m$ set

$$
\Omega_{k, i}:=\left\{-(K+k)<\frac{\log \left|u_{i}\right|}{\alpha_{i}}<1-(K+k)\right\} .
$$

Then we can estimate the volume of $\pi^{\text {an }}\left(\Omega_{k}\right)$ as follows:

$$
\begin{aligned}
& \operatorname{Vol} \pi^{\mathrm{an}}\left(\Omega_{k}\right)=(\sqrt{-1})^{n} \int_{\pi^{\mathrm{an}}\left(\Omega_{k}\right)} \eta \wedge \bar{\eta}=(\sqrt{-1})^{n} \int_{\Omega_{k}}\left|J \pi^{\mathrm{an}}\right|^{2} \eta_{u} \wedge \bar{\eta}_{u} \\
& \gtrsim \prod_{i=1}^{m} \sqrt{-1} \int_{\Omega_{k, i}}\left|u_{i}\right|^{2 A_{i}-2} d u_{i} \wedge d \bar{u}_{i} \gtrsim \prod_{i=1}^{m} \exp \left(-k\left(2 A_{i} \alpha_{i}\right)\right)=\exp (-2 k A(v)) .
\end{aligned}
$$

This estimate together with 4.6 concludes the proof of Theorem D'. By choosing $\mathfrak{q}=\mathcal{O}_{U}$ throughout all the arguments (see also Remark 2.4), we also obtain a proof of Theorem D. 


\section{REFERENCES}

[Ber06] B. Berndtsson. Subharmonicity properties of the Bergman kernel and some other functions associated to pseudoconvex domains. Ann. Inst. Fourier 56 (2006), 16331662. 4

[BFJ08] S. Boucksom, C. Favre and M. Jonsson. Valuations and plurisubharmonic singularities. Publ. Res. Inst. Math. Sci. 44 (2008), 449-494. 419

[Dem] J.-P. Demailly. Complex analytic and algebraic geometry. Book available at www-fourier.ujf-grenoble.fr/ demailly. 13

[Dem87] J.-P. Demailly. Nombres de Lelong généralisés, théorèmes d'intégralité et d'analyticité. Acta Math. 157 (1987), 153-169. 1, 14, 16

[Dem92] J.-P. Demailly. Regularization of closed positive currents and intersection theory. J. Alg. Geom. 1 (1992), 361-409. 3, 19

[Dem93] J.-P. Demailly. A numerical criterion for very ample line bundles. J. Differential Geom. 37 (1993), 323-374. 3, 19

[DEL00] J.-P. Demailly, L. Ein and R. Lazarsfeld. A subadditivity property of multiplier ideals. Michigan Math. J. 48 (2000), 137-156. 3, 19

[DK01] J.-P. Demailly and J. Kollár. Semicontinuity of complex singularity exponents and Kähler-Einstein metrics on Fano orbifolds. Ann. Sci. École Norm. Sup. (4) 34 (2001), 525-556. 1, 2, 13, 14, 18, 19, 20,

[ELS03] L. Ein, R. Lazarsfeld, and K. E. Smith, Uniform approximation of Abhyankar valuations in smooth function fields, Amer. J. Math. 125 (2003), 409-440. 5

[ELSV04] L. Ein, R. Lazarsfeld, K. E. Smith and D. Varolin. Jumping coefficients of multiplier ideals. Duke Math. J. 123 (2004), 469-506. 3, 6, 14

[FJ04] C. Favre and M. Jonsson. The valuative tree. Lecture Notes in Mathematics 1853. Springer-Verlag, Berlin, 2004. 4

[FJ05a] C. Favre and M. Jonsson. Valuative analysis of planar plurisubharmonic functions. Invent. Math. 162 (2005), 271-311. 4

[FJ05b] C. Favre and M. Jonsson. Valuations and multiplier ideals. J. Amer. Math. Soc. 18 (2005), 655-684. 2, 4,8

[Gue10] H. Guenancia. Toric plurisubharmonic functions and analytic adjoint ideal sheaves. arXiv:1011.3162. 4

[Hor94] L. Hörmander. Notions of convexity. Progress in Mathematics, 127. Birkhäuser, Boston, MA, 1994. 13

[Hu12a] Z. Hu. Valuative multiplier ideals. Preprint. 8

[Hu12b] Z. Hu. Valuations and log canonical thresholds. Preprint. 8

[Jon12] M. Jonsson. Dynamics on Berkovich spaces in low dimensions. arXiv:1201.1944. To appear in Berkovich spaces and applications, Séminaires et Congrès, Société Mathématique de France. 4

[JM12] M. Jonsson and M. Mustaţă. Valuations and asymptotic invariants for sequences of ideals. Ann. Inst. Fourier 62 (2012), 2145-2209. 2, 3, 4, 5, 6, 7, 8, 9, 10, 11, 23

[Kis87] C.-O. Kiselman. Un nombre de Lelong raffiné. In Séminaire d'analyse complexe et géométrie 1985-1987. Faculté des sciences de Tunis et Faculté des Sciences et Techniques de Monastir, 1987, pp. 61-70. 1, 4, 14, 16

[Kis94] C. O. Kiselman. Attenuating the singularities of plurisubharmonic functions. Ann. Polon. Math. 60 (1994), 173-197. 1, 4, 14, 15,16

[Lag10] A. Lagerberg. A new generalization of the Lelong number. arXiv:1001.3562. 4

[Laz] R. Lazarsfeld. Positivity in algebraic geometry II. Ergebnisse der Mathematik und ihrer Grenzgebiete, 3. Folge, Vol. 49, Springer-Verlag, Berlin, 2004. 6

[Lel69] P. Lelong. Plurisubharmonic functions and positive differential forms. Gordon and Breach, New York, and Dunod, Paris, 1969. 1, 4, 16

[Mat80] H. Matsumura. Commutative algebra. Mathematics Lecture Note Series, 56. Benjamin/Cummings. Reading, Mass., 1980. 3, 11, 23 
[Mus02] M. Mustaţă. On multiplicities of graded sequences of ideals. J. Algebra 256 (2002), 229-249. 6

[Nad89] A. M. Nadel. Multiplier ideal sheaves and existence of Kähler-Einstein metrics of positive scalar curvature. Proc. Nat. Acad. Sci. USA 86 (1989), 7299-7300. 18

[Nad90] A. M. Nadel. Multiplier ideal sheaves and Kähler-Einstein metrics of positive scalar curvature. Ann. of Math. 132 (1990), 549-596. 18

[Ras06] A. Rashkovskii. Relative types and extremal problems for plurisubharmonic functions. Int. Math. Res. Not. 2006, Art. ID 76283. 4

[Siu74] Y.-T. Siu. Analyticity of sets associated to Lelong numbers and the extension of positive closed currents. Inventiones. Math. 27 (1974), 53-156. 1

[Sko72] H. Skoda. Sous-ensembles analytiques d'ordre fini ou infini dans $\mathbf{C}^{n}$. Bull. Soc. Math. France 100 (1972), 353-408. 1

Dept of Mathematics, University of Michigan, Ann Arbor, Mi 48109-1043, USA

E-mail address: mattiasj@umich.edu, mmustata@umich.edu 